\title{
A multidimensional discontinuous Galerkin modeling framework for overland flow and channel routing
}

\author{
Dustin W West ${ }^{\mathrm{a}}$, Ethan J Kubatko ${ }^{\mathrm{b}, *}$, Colton J Conroy ${ }^{\mathrm{c}}$, Mariah Yaufman ${ }^{\mathrm{d}}$, \\ Dylan Wood ${ }^{\mathrm{b}}$ \\ ${ }^{a}$ Hazen and Sawyer, 150 E. Campus View Boulevard, Suite 133, Columbus, OH, USA \\ ${ }^{b}$ Department of Civil, Environmental and Geodetic Engineering, The Ohio State University, \\ Columbus, OH, USA \\ ${ }^{c}$ Columbia University, Applied Physics and Applied Mathematics Department, New York \\ City, NY, USA \\ ${ }^{d}$ EMH $\& 3$ T Inc., 5500 New Albany Road, New Albany, OH, USA
}

\begin{abstract}
In this paper, we present the development and application of a new multidimensional, unstructured-mesh model for simulating coupled overland/openchannel flows in the kinematic wave approximation regime. The modeling approach makes use of discontinuous Galerkin (DG) finite element spatial discretizations of variable polynomial degree $p$, paired with explicit Runge-Kutta time steppers, and is supported by advancements made to an automatic mesh generation tool, ADMESH+, that is used to construct constrained triangulations for channel routing. The developed modeling framework is applied to a set of four test cases, where numerical results are found to compare well with known analytic solutions, experimental data and results from another well-established (structured, finite difference) model within the area of application. The numerical results obtained demonstrate the accuracy and robustness of the developed modeling framework and highlight the potential benefits of using $p$ (polynomial) refinement for hydrological simulations.
\end{abstract}

Keywords: overland flow, open-channel flow, discontinuous Galerkin, finite element, mesh generation

*Corresponding author 


\section{Introduction}

Physically-based (or synonymously process-based) watershed modeling in hydrology has a history of roughly five decades; see the recent review articles $[1,2]$. At a very broad level, it seeks to describe the movement of water throughout the hydrologic cycle by means of the basic conservation laws of fluid mechanics and thermodynamics [3]. Comprehensively (and more specifically), it involves modeling the flow and transport of water through the atmosphere, over the (land) surface and through the subsurface using conservation (or balance) equations of mass, momentum and energy, with relevant atmosphere-land and surface-subsurface interface and boundary conditions. Given the multi-scale and multi-physics nature of these varied hydrological components, the complex coupling mechanisms that exist between them and a number of other factors (see, for example, [1]), development of robust and efficient physically-based models for watershed hydrology remains a challenge [2].

This challenge exists at both the system- and component-level development of hydrological models; e.g., the surface flow component of the hydrological system alone, which is the focus of the present work, exhibits many of the challenging features found at the system level, such as widely varying scales, multiple physical processes and coupled (sub)components. This leads directly to the consideration of a number of (interrelated) model development questions regarding, for example, governing equations and related parameterizations used, geometric representations employed (e.g., structured or unstructured domain discretization, dimensionality of the various watershed features) and numerical methods implemented. Ideally, the developed modeling framework should offer a certain degree of flexibility in addressing these questions and be in a position to continue to develop and evolve, allowing model developers and users, for example, to accommodate new high-resolution data sets with relative ease, take advantage of ever-evolving computational platforms and be able to couple their models to other model components of the larger hydrological system.

In this paper, we present the development and application of a new sur- 
face flow hydrological modeling framework for simulating coupled overland and open-channel flow that is designed to address these points and meet some of the challenges posed by hydrological modeling. The modeling framework makes use of discontinuous Galerkin (DG) methods and mesh generation procedures designed specifically for the developed approach. DG methods appear to be an especially judicious choice as the numerical basis for hydrological models on several fronts. First, as a class of finite element methods, DG methods can make use of unstructured meshes to represent the complex geometry typical of watersheds in an accurate and computationally efficient manner. This is particularly the case for the specific multidimensional approach considered in this work (and discussed in more detail below). Second, with numerical solutions based on piecewise polynomial spaces that allow discontinuities to occur between elements, DG methods can also easily support the use of polynomial $(p)$ refinement or enrichment as an efficient means to obtain highly accurate solutions, as is demonstrated in the numerical results presented in this paper, and to support the use of high-order interpolants in data-rich settings. Additionally, the methods have been shown to be highly parallelizable in implementations on both traditional parallel platforms $[4,5]$ and graphics-processing units (GPUs) $[6,7,8]$. And finally, DG methods possess the important property of local (element-wise) conservation, which is of fundamental importance in hydrological modeling, especially when coupling various model components $[2,9]$.

In developing this modeling framework, we took, as a simple starting point, the conceptual model of a drainage basin (equivalently watershed or catchment) presented in [10] described as consisting of "two geomorphological components: a set of hillslopes dominated by unconfined overland flow and a branching network of channels conveying concentrated flow." This conceptual model is actually very useful from a computational modeling perspective in that the division of the drainage basin into two distinct (albeit coupled) geomorphological components correspondingly points to the use of two distinct modeling approaches to represent them, in terms of describing both their geometry and the associated flow. 
With respect to geometric representations, the latter geomorphological component (the channel network) is most naturally represented as a one-dimensional, tree-like structure, while the "unconfined" nature of the overland flow component points to the use of a two-dimensional representation that allows for a certain degree of flexibility in representing the geometry given both the geometric complexity of a typical drainage basin and the coupled nature of the two geomorphological components. In the model developed here, we make use of constrained meshes or triangulations, wherein the line segments composing the channel network are forced into the triangulation as element edges (but are otherwise unstructured); see, for example, Figures 5 and 6 . (We note that the one-dimensional domain discretizations are also "unstructured" in the sense that variable-length one-dimensional elements can be used along the channel networks.) Part of the challenge in implementing and applying such an approach is the construction of high-quality, constrained unstructured meshes, which is by no means trivial. This challenge is addressed in the present work through the continued development of an automatic mesh generation platform, called Admesh+, developed by the first three authors [11] and discussed in Section 3.3 of this paper.

With respect to the associated flows, we first note that the "unconfined" and "concentrated" surface flows of these two geomorphological components are both well described by the (depth-averaged) two-dimensional shallow water equations but can, in many situations, be modeled with sufficient accuracy using a number of simplified equations derived from them. First, for example, given the decision to represent the channel network component in one-dimension, the associated flow can be described adequately using the section-averaged, onedimensional shallow water (or Saint-Venant) equations. Moreover, based on the relative magnitude of the terms appearing in either the one- or two-dimensional shallow water equations in a given flow scenario, various approximations of a simpler nature can be applied. These approximations include, for example, the so-called gravity, diffusion and kinematic wave equations, the solutions of which have been shown to adequately describe the flow under proper sets of conditions 
(see, for example, [12], which provides a nice summary and characterization of these conditions).

The simplest of the these approximations, the kinematic wave approximation, has been found to be "accurate enough" or "reasonably accurate" (see [13] and also [14] and the references therein) under the natural conditions occurring in many drainage basins and is chosen as the basis for the developed model. Use of the kinematic wave approximations is appealing from both a conceptual and numerical perspective, in that both the overland and open-channel flows can be described using single hyperbolic equations (rather than systems of equations). Thus, DG methods are a natural choice given the great success they have demonstrated for the solution of hyperbolic conservation laws in a wide variety of fields (see, for example, $[15,16,17,18,19]$ ), though not, it is to be noted, in the present context of kinematic approximations for open-channel and overland flow. We also note that our decision to employ kinematic wave approximations is dictated, in part, by the viewpoint that this work represents the first step in the development of such a (DG-based) model, starting with (essentially) the simplest approximation in order to investigate both the merits and limitations of such an approach (which, to a certain extent, are revealed in the test cases considered in Section 4.1 of this paper).

The rest of this paper is organized as follows. In the next section, we first present the (depth-averaged) two- and (section-averaged) one-dimensional shallow water equations, insomuch that their corresponding kinematic wave approximations - the governing equations of the model — can be introduced in context. This is followed by a description of the discretization techniques employed by the model, which consist of the multidimensional domain discretization approach introduced above, the one- and two-dimensional DG spatial discretizations and a set of explicit Runge-Kutta (RK) discretizations to advance the model in time. In Section 3.3, we outline the specific steps involved in and the computational tools employed for generating constrained computational meshes (i.e., domain discretizations) for the developed modeling approach. Next, results from a set of four test cases are presented in Section 4.1, where numerical solutions are 
compared to known analytic solutions, experimental data and numerical results from another (well-established) model in the field. And finally, to conclude, we provide a brief summary of the work and discuss our ongoing and future work in this area.

\section{Governing equations}

\subsection{Overland flow}

Using a Cartesian coordinate system $(x, y, z)$, consider a land surface $z=$ $f(x, y)$ that describes the topography of a given watershed. When this land surface is subjected to a rainfall rate $r(x, y, t)$, which (in general) will be a function of both the horizontal $(x, y)$ coordinates and time $t$, that exceeds its infiltration rate $i(x, y, t)$, flow over the land surface - known as surface runoff or overland flow (the latter term will be used here) - can occur. This type of flow can be described by the two-dimensional shallow water equations, which consist of (i) a depth-integrated continuity equation

$$
\frac{\partial H}{\partial t}+\nabla \cdot H \mathbf{U}=r-i,
$$

where $H(x, y, t)$ is the depth of water and $\mathbf{U}=(u(x, y, t), v(x, y, t))$ is the depthaveraged, horizontal velocity vector; and (ii) momentum balance equations in the horizontal directions, which can be written in the form

$$
\frac{\partial}{\partial t}(H \mathbf{U})+\nabla \cdot \mathbf{F}=g H\left(\mathbf{S}_{0}-\mathbf{S}_{f}\right)
$$

141 where

$$
\mathbf{F}=\left(\begin{array}{cc}
H u^{2}+\frac{1}{2} g h^{2} & H u v \\
H u v & H v^{2}+\frac{1}{2} g H^{2}
\end{array}\right), \quad \mathbf{S}_{0}=\left(\begin{array}{c}
\frac{\partial z}{\partial x} \\
\frac{\partial z}{\partial y}
\end{array}\right), \quad \mathbf{S}_{f}=\left(\begin{array}{c}
S_{f_{x}} \\
S_{f_{y}}
\end{array}\right),
$$

with $g$ being gravitational acceleration and the vectors $\mathbf{S}_{0}$ and $\mathbf{S}_{f}$ being the bed (land) slope and friction slope terms, respectively. The latter is typically computed using a Manning-type resistance law,

$$
\mathbf{S}_{f}=\frac{n^{2}\|\mathbf{U}\|}{H^{4 / 3}} \mathbf{U}
$$


where $n$ is the Manning resistance coefficient, which is dependent on the land surface roughness and can be obtained from standard references, see, for example, $[20]$.

\subsection{Open-channel flow}

Overland flow often collects into natural and man-made channels that act to route the water within a given watershed to a single converging point. The resulting (open-channel) flow can be described using one-dimensional, sectionaveraged versions of the equations given above (also known as the Saint-Venant equations). These equations can be written in the form

$$
\frac{\partial A}{\partial t}+\frac{\partial Q}{\partial s}=q_{L}
$$

$$
\frac{\partial Q}{\partial t}+\frac{\partial}{\partial s}\left(\frac{Q^{2}}{A}+g I_{1}\right)=g A\left(S_{0}-S_{f}\right)+g I_{2},
$$

where $s$ is a curvilinear coordinate along the river or channel centerline, $A$ denotes the cross-sectional area of flow, $Q=\bar{u} A$ is the mean cross-sectional volumetric discharge (note: $\bar{u}$ is the section-averaged velocity), $q_{L}$ is a lateral inflow rate per unit length of channel, and $I_{1}$ and $I_{2}$ are integral terms accounting for pressure forces (hydrostatic and wall pressure terms, respectively).

In the case of open-channel flow, a Manning formulation for the friction slope $S_{f}$ takes the form

$$
S_{f}=\frac{n \bar{u}^{2}}{R_{h}{ }^{2 / 3}},
$$

where $R_{h}=A / P$ is the hydraulic radius, which is a ratio of the cross-sectional area of flow $A$ and the wetted perimeter $P$.

\subsection{The kinematic wave approximation}

In many cases of both open-channel and overland flow, the friction and bedslope terms are dominant in the momentum equations (i.e., the remaining terms are negligibly small). This leads to, for example, in the case of open-channel flow, an approximate relationship of the form

$$
S_{0} \approx S_{f}=\frac{n \bar{u}^{2}}{R_{h}{ }^{2 / 3}} .
$$


Solving this expression for $\bar{u}$ gives an approximation for the velocity of the form

$$
\bar{u} \approx \alpha \sqrt{S_{0}} R_{h}^{\beta}
$$

where $\alpha=1 / n$ and $\beta=2 / 3$. (Note: setting $\alpha=C$, the Chezy coefficient, and $\beta=1 / 2$ in (2) gives the well-known Chezy formula).

Substitution of (2) into (1) results in the so-called kinematic wave approximation for open-channel flow, which reduces the problem to a single equation in terms of a single unknown $A$; that is,

Kinematic wave approximation: $1 D$ open-channel flow

$$
\frac{\partial A}{\partial t}+\frac{\partial}{\partial s} \underbrace{\left(\alpha \sqrt{S_{0}} R_{h}{ }^{\beta} A\right)}_{=Q}=q_{L} .
$$

Similar considerations in the case of overland flow result in the following kinematic wave approximation (when using a Manning-type resistance law)

Kinematic wave approximation: 2D overland flow

$$
\frac{\partial H}{\partial t}+\nabla \cdot \mathbf{q}=r-i
$$

where $\mathbf{q}=\left(q_{x}, q_{y}\right)$ with

$$
q_{x}=\frac{S_{0 x}}{n \sqrt{S_{0}}} H^{5 / 3}, \quad q_{y}=\frac{S_{0 y}}{n \sqrt{S_{0}}} H^{5 / 3}
$$

where $S_{0_{x}}$ and $S_{0_{y}}$ are the $x$ and $y$ components, respectively, of the slope vector $\mathbf{S}_{0}$; see, for example, [21] for a more detailed exposition on this equation. Note that the equations are coupled via the discharge terms $q_{L}$ and $\mathbf{q}$ of Equations (3) and (4), respectively, which is described in more detail for our modeling approach in subsection 3.2.1. 


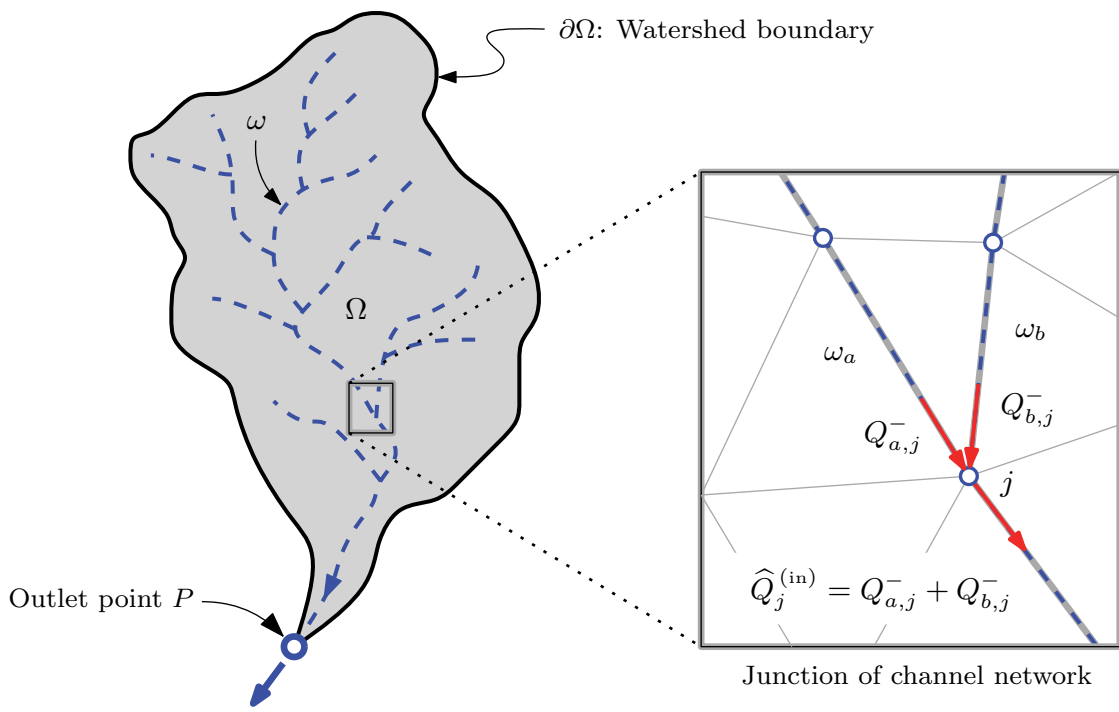

Figure 1: Simple schematic of a watershed of horizontal extent $\Omega$, with boundary $\partial \Omega$ indicated by the thick black line. In hydrologic modeling, the watershed's drainage network (the set of dashed blue lines denoted by $\omega$ ) is typically represented as a set of connected line segments $\omega_{i}$. The inset (right) shows a simple example of how junctions are handled in the numerical model; see discussion in Section 3.2.

\section{Discretization}

\subsection{Domain discretization}

Consider a watershed, or drainage basin, with horizontal extent $\Omega$ as illustrated in Figure 1. By definition (of a watershed), all surface water flow within $\Omega$ will converge to a single outlet point $P$. The outlet point is the terminus of the watershed's drainage network - a tree-like structure of "concentrated" (open-channel) flow pathways that collect the "unconfined" overland flow of neighboring hillslopes. This description gives rise to the conceptual model of a drainage basin presented earlier that consists of "two geomorphological components: a set of hillslopes dominated by unconfined overland flow and a branching network of channels conveying concentrated flow" [10].

Given the relative scales of these two components, in hydrological modeling 
the latter component (the channel network) is typically represented simply as a set of connected line segments, which we denote by $\omega_{i}$; see inset of Figure 1. This indicates that the set of hillslopes that constitute (what we will refer to as) the overland flow component completely covers the watershed. Therefore, in our modeling approach, the kinematic wave approximation for overland flow, i.e., Eq. (4), is solved over the entire domain $\Omega$, while the kinematic wave approximation for open-channel flow, i.e., Eq. (3), is solved over the (one-dimensional) domain $\omega$.

Discretization of the watershed needs to take into account the coupled nature of the two geomorphological components. This is accomplished in the present modeling approach through the use of a finite element partition, or mesh, $\mathcal{T}_{h}$ of $\Omega$, consisting of elements $\Omega_{j}$ with edges aligned along $\omega$; see, as examples, Figures 5 and 6 . The collection of edges aligned along $\omega$ form a one-dimensional finite element partition $\mathcal{E}_{h}$ of $\omega$, consisting of channel elements $\omega_{i}$. The construction of such a mesh, starting from a given digital elevation model (DEM), is described in detail in Section 3.3.

\subsection{The discontinuous Galerkin spatial discretizations}

Given finite element partitions as described above, we obtain weak forms of (3) and (4) by multiplying the equations by suitably smooth test functions $v(s)$ and $w(x, y)$ and integrating over each one-dimensional $\left(\omega_{i} \in \mathcal{E}_{h}\right)$ and twodimensional $\left(\Omega_{j} \in \mathcal{T}_{h}\right)$ element, respectively, where the flux terms are integrated by parts. This yields

$$
\int_{\omega_{i}} \frac{d A}{d t} v d s-\int_{\omega_{i}} Q \frac{d v}{d s} d s+\left.Q v\right|_{\partial \omega_{i}}=\int_{\omega_{i}} q_{L} v d s
$$

$$
\int_{\Omega_{j}} \frac{d H}{d t} w d \mathbf{x}-\int_{\Omega_{j}} \mathbf{q} \cdot \nabla w d \mathbf{x}+\int_{\partial \Omega_{j}} \mathbf{q} \cdot \mathbf{n} w d s=\int_{\Omega_{j}}(r-i) w d \mathbf{x},
$$

where $d \mathbf{x}=d x d y, \partial \omega_{i}$ and $\partial \Omega_{j}$ indicate the boundaries of $\omega_{i}$ and $\Omega_{j}$, respectively, and $\mathbf{n}$ is the outward unit normal to the element boundary $\partial \Omega_{j}$.

Next, we seek approximate (trial) solutions $\left(A_{h}, H_{h}\right)$ to $(A, H)$ that belong 
to the finite-dimensional spaces

$$
\begin{aligned}
\mathbb{V}_{h p}(\omega) & =\left\{v:\left.v\right|_{\omega_{i}} \in \mathbb{P}_{p}\left(\omega_{i}\right), \forall \omega_{i} \in \mathcal{E}_{h}\right\} \\
\mathbb{W}_{h p}(\Omega) & =\left\{w:\left.w\right|_{\Omega_{j}} \in \mathbb{Q}_{p}\left(\Omega_{j}\right), \forall \Omega_{j} \in \mathcal{T}_{h}\right\},
\end{aligned}
$$

222

respectively, where $\mathbb{P}_{p}$ denotes the space of polynomials of degree (at most) $p$ and $\mathbb{Q}_{p}$ denotes the space of bipolynomials complete up to (at most) degree $p$.

Note that the spaces defined above do not enforce global continuity of the functions. Therefore, the trial solutions may be discontinuous at element boundaries. Consider, for example, a discontinuity in $A_{h}$ at a (non-junction) point $s_{j}$ with values

$$
A_{j}^{-}=\lim _{s_{j} \rightarrow s_{j}^{-}} A_{h}\left(s_{j}, t\right) \text { and } A_{j}^{+}=\lim _{s_{j} \rightarrow s_{j}^{+}} A_{h}\left(s_{j}, t\right)
$$

as $s_{j}$ is approached from the upstream and downstream element, respectively. This discontinuity in $A_{h}$, in turn, introduces, dual values of the flux $Q_{j}^{-}=$ $Q\left(A_{j}^{-}\right)$and $Q_{j}^{+}=Q\left(A_{j}^{+}\right)$at point $s_{j}$. In the DG formulation, we replace these (possibly) dual-valued fluxes (again, at non-junction points) with single-valued numerical fluxes $\widehat{Q}_{j}$. Given that kinematic waves propagate downstream only, we can simply employ an upstream numerical flux, that is, $\widehat{Q}_{j} \equiv Q_{j}^{-}$. Similarly, along a given flow edge $\Gamma_{i}$ in the two-dimensional formulation, we replace $\left.\mathbf{q}\right|_{\Gamma_{i}}$ by $\widehat{\mathbf{q}}=\mathbf{q}\left(H_{i}^{-}\right)$, where $H_{i}^{-}$is the upstream trace of $H_{h}$ along edge $\Gamma_{i}$.

In the one-dimensional formulation, note that there may be multiple upstream elements at junction points $s_{j}$, leading to multiple flux values $Q_{i, j}^{-}$at point $s_{j}$ from the multiple upstream elements $\omega_{i}$. Therefore, in the downstream element, we take the incoming numerical flux to be

$$
\widehat{Q}_{j}^{(\text {in })}=\sum_{i} Q_{i, j}^{-} \quad(\text { In downstream elements of junctions }),
$$

where the sum is over all upstream elements $\omega_{i}$ connected at point $j$. (Note, however, that the outgoing numerical flux used in a given upstream element $\omega_{i}$ is simply $\widehat{Q}_{j}=Q_{i, j}^{-}$as before.) The inset of Figure 1 presents a simple example of this approach for a junction with two upstream elements. (We note that the 
channel network is an arborescence, rooted at the outlet point of the drainage basin; see, for example, [22]. In the present context, this indicates that there would not be more than one downstream element at a junction point.)

Replacing $(A, H)$ by their trial solutions $\left(A_{h}, H_{h}\right)$, using the numerical fluxes $(\widehat{Q}, \widehat{\mathbf{q}})$ for $(Q, \mathbf{q})$ at the element boundaries as defined above, and taking the test functions $(v, w)=\left(v_{h}, w_{h}\right)$, we arrive at the following discrete weak form of the problem: find $A_{h} \in \mathbb{V}_{h p}$ and $H_{h} \in \mathbb{W}_{h p}$ such that for all test functions $v_{h} \in \mathbb{V}_{h p}$ and $w_{h} \in \mathbb{W}_{h p}$

$$
\int_{\omega_{i}} \frac{d A_{h}}{d t} v_{h} d s-\int_{\omega_{i}} Q\left(A_{h}\right) \frac{d v_{h}}{d s} d s+\left.\widehat{Q} v_{h}^{\text {(int) }}\right|_{\partial \omega_{i}}=\int_{\omega_{i}} q_{L} v_{h} d s
$$

$\int_{\Omega_{j}} \frac{d H_{h}}{d t} w_{h} d \mathbf{x}-\int_{\Omega_{j}} \mathbf{q}\left(H_{h}\right) \cdot \nabla w_{h} d \mathbf{x}+\int_{\partial \Omega_{j}} \widehat{\mathbf{q}} \cdot \mathbf{n} w_{h}^{(\mathrm{int})} d s=\int_{\Omega_{j}}(r-f) w_{h} d \mathbf{x}$, over each $\omega_{i} \in \mathcal{E}_{h}$ and each $\Omega_{j} \in \mathcal{T}_{h}$. (Note: $v_{h}^{(\mathrm{int})}$ and $w_{h}^{(\mathrm{int})}$ denote the interior traces of the test functions.)

Upon choosing bases for the finite element spaces $\mathbb{V}_{h p}$ and $\mathbb{W}_{h p}$, the above discrete weak forms reduce to systems of time-dependent ordinary differential equations (ODEs) of the form

$$
\frac{d \mathbf{A}}{d t}=\mathcal{R}_{h p}^{(1 \mathrm{D})}(\mathbf{A}), \quad \frac{d \mathbf{H}}{d t}=\mathcal{R}_{h p}^{(2 \mathrm{D})}(\mathbf{H}),
$$

where $\mathbf{A}$ and $\mathbf{H}$ are vectors of time-dependent degrees of freedom associated with $A_{h}$ and $H_{h}$, respectively, and $\mathcal{R}_{h p}^{(\mathrm{nD})}$ is the $n$-dimensional DG spatial operator.

As an example, in the one-dimensional case, given a set of basis functions $\mathbf{\Phi}=\left[\phi_{0}, \phi_{1}, \ldots, \phi_{p}\right]^{\mathrm{T}}$ for the space $\mathbb{V}_{h p}$, the trial solution $A_{h}$ over $\omega_{i}$ can be expressed as

$$
\left.A_{h}\right|_{\omega_{i}}=\boldsymbol{\Phi}^{\mathrm{T}} \mathbf{A}_{i}
$$

where $\mathbf{A}_{i}$ is a (column) vector of the time-dependent degrees of freedom of the DG solution over $\omega_{i}$. The discrete weak form for each $\omega_{i}$ can then be written as a system of ODEs in the form

$$
\mathbf{M}_{i} \frac{d \mathbf{A}_{i}}{d t}=\mathbf{F}_{i},
$$


where the components, $M_{k l}$, of the element mass matrix $\mathbf{M}_{i}$ are given by $M_{k l}=$ $\int_{\omega_{i}} \phi_{k} \phi_{l} d x$ and

$$
\mathbf{F}_{i}=\left[F_{i}\left(\phi_{0}\right), F_{i}\left(\phi_{1}\right), \ldots, F_{i}\left(\phi_{p}\right)\right]^{\mathrm{T}}
$$

with

$$
F_{i}\left(\phi_{k}\right)=\int_{\Omega_{i}} Q \frac{d \phi_{k}}{d s} d s-\left.\widehat{Q} \phi_{k}^{(\mathrm{int})}\right|_{\partial \omega_{i}}
$$

We note that $\mathbf{M}_{i}$ is a local (element) matrix and that the elements are coupled through the numerical flux terms appearing in $\mathbf{F}_{i}$.

Inverting the element mass matrices, the full set of one-dimensional semidiscrete equations over a set of $N$ elements can be written compactly as

$$
\frac{d \mathbf{A}}{d t}=\mathcal{R}_{h p}^{(1 \mathrm{D})}(\mathbf{A})
$$

where

$$
\mathbf{A}=\left(\begin{array}{c}
\mathbf{A}_{1} \\
\mathbf{A}_{2} \\
\vdots \\
\mathbf{A}_{N}
\end{array}\right), \quad \mathcal{R}_{h p}^{(1 \mathrm{D})}(\mathbf{A})=\left(\begin{array}{c}
\mathbf{M}_{1}^{-1} \mathbf{F}_{1} \\
\mathbf{M}_{2}^{-1} \mathbf{F}_{2} \\
\vdots \\
\mathbf{M}_{N}^{-1} \mathbf{F}_{N}
\end{array}\right)
$$

A similar set of equations is obtained in the two-dimensional case.

In our implementation, we use the set of Legendre polynomials of degree $p$ as our basis for $\mathbb{V}_{h p}$ and either a tensor product of Legendre polynomials, in the case of quadrilateral elements, or the so-called Dubiner basis, in the case of triangular elements, for $\mathbb{W}_{h p}$; see, for example, [23], for more details on these bases. We note that these are so-called modal bases that are hierarchical; for example, with the Dubiner basis, a first-order approximation is obtained by using one basis function, $\phi_{0}=1$, a second-order approximation by adding two additional basis functions, $\phi_{1}=(3 y+1) / 2$ and $\phi_{2}=x+(y+1) / 2$, etc. All integrals of the DG spatial operators are computed using Gauss quadrature of sufficient degree.

\subsubsection{Multidimensional coupling of the spatial discretizations}

The one- and two-dimensional DG equations are coupled through the lateral flux terms $q_{L}$. Specifically, for a given pair of two-dimensional elements $\Omega_{j}$ and 


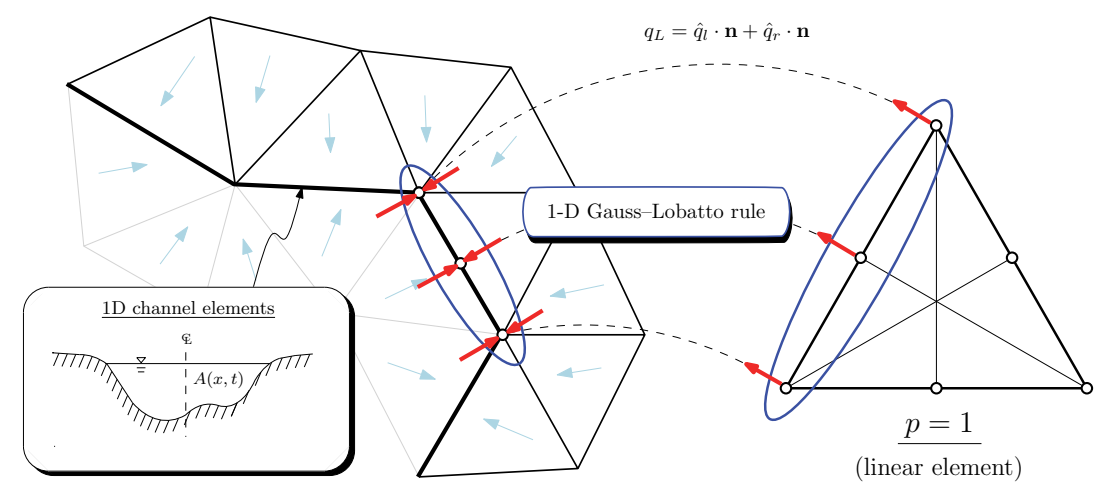

Figure 2: An illustration of the multidimensional coupling that is accomplished by simply setting $q_{L}=\widehat{q} \cdot \mathbf{n}$ at the common $1 \mathrm{D}$ and 2D (edge) Gauss-Lobatto integration points.

$\Omega_{j^{\prime}}$ with "left" $(l)$ and "right" $(r)$ edges, $\Gamma_{l}$ and $\Gamma_{r}$, that are coincident with channel element $\omega_{i}$, we must have

$$
\int_{\omega_{i}} q_{L} d s=\int_{\Gamma_{l}} \widehat{q} \cdot \mathbf{n} d s+\int_{\Gamma_{r}} \widehat{q} \cdot \mathbf{n} d s .
$$

This condition is enforced numerically by using the same set of Gauss quadrature points for numerically integrating the one-dimensional channel elements $\omega_{i}$ and the edge integrals of the the two-dimensional overland flow elements. Then, we simply set $q_{L}=\widehat{q}_{l} \cdot \mathbf{n}+\widehat{q}_{r} \cdot \mathbf{n}$ at a given Gauss point. Here, we make use of Gauss-Lobatto rules, which include the endpoints of the interval, of degree $2 p+1$ for a DG spatial discretization of degree $p$, as suggested in [24]; see Figure 2 for an illustration of this idea for $p=1$ (linear) elements.

\subsection{Runge-Kutta Time Discretization}

The systems of ODEs given by (5) are solved in time using explicit, strongstability-preserving (SSP) Runge-Kutta (RK) time steppers of order $p+1$, where $p$ is the degree of the DG spatial approximation. Application of an $s$-stage SSP RK method to, for example, the one-dimensional system of ODEs using a time 
step of $\Delta t$ can be written in the following Shu-Osher representation

$$
\begin{aligned}
\mathbf{A}^{(0)} & =\mathbf{A}^{n} \\
\mathbf{A}^{(i)} & =\sum_{l=0}^{i-1} \alpha_{i l} \mathbf{A}^{(l)}+\Delta t \beta_{i l} \mathcal{R}_{h p}^{(1 \mathrm{D})}\left(\mathbf{A}^{(l)}\right), \quad i=1,2, \ldots, s \\
\mathbf{A}^{n+1} & =\mathbf{A}^{(s)},
\end{aligned}
$$

where $\mathbf{A}^{n} \equiv \mathbf{A}(n \Delta t), n \in \mathbb{N}$, and where $\alpha_{i l}$ and $\beta_{i l}$ are coefficients satisfying certain order, consistency and SSP constraints; see, for example, [25]. We note that minmod-based slope limiters are applied at each RK stage to prevent spurious oscillations; see, for example, [24].

\section{Mesh generation for the multidimensional approach}

Starting from a DEM of a given geographical area, the generation of computational meshes for the numerical approach outlined above is carried out in two main steps:

1. A DEM data-extraction step that makes use of a set of open-source Matlab functions called TopoToolbox [26] to pre-process (e.g., pit-fill, smooth, etc) the DEM data and automatically extract watershed boundaries and channel networks. Figure 3, for example, shows the extracted watershed boundary and channel network obtained from a sample DEM (see also Figure 17 of Section 4.1). Various options are available for identifying the extent of the channel network in TopoToolbox; e.g., selection can be made based on the Strahler stream order or calculated flow accumulation; see [26] for details.

2. A mesh-generation step that makes use of the extracted DEM data and a program developed by the first three authors, called ADMESH+ [11], which has been further developed under this work. This step ultimately provides the underlying mesh data structure that is required by the outlined numerical approach and that is described in detail below. 


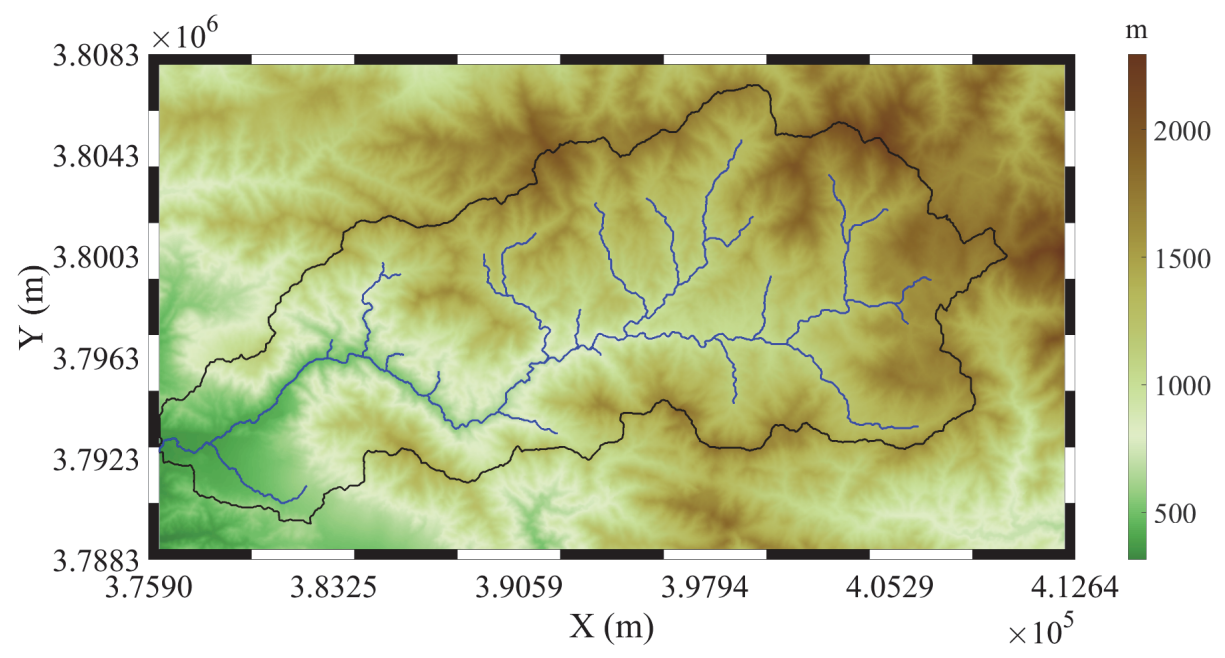

Figure 3: The channel network (blue lines) and watershed boundary (black lines) extracted from a sample DEM using TopoToolbox.

\subsection{Admesh+: Generating a constrained mesh and the accompanying data structure}

A full description of the ADMEsH+ methodology can be found in [11], which incorporates and builds on the ideas and methodology of Persson's DistMesh program - a simple, open-source mesh generator implemented in Matlab [27]. Briefly, a mesh- or element-size function, $h(\mathbf{x})$, is first constructed that is used to prescribe element sizes $h$ throughout the domain. These sizes are based on a number of geometric factors, such as shoreline/boundary curvature and bathymetric/topographic gradients, as well as user-defined inputs, such as target maximum/minimum element sizes and mesh-grading specifications (i.e., the ratio of neighboring elements should not exceed some specified factor). Given the element-size function, a Delaunay triangulation of an initial set of mesh nodes with a density proportional to $1 / h(x)^{2}$ is then generated and the nodes of this initial mesh are re-positioned by solving for force equilibrium (iteratively) around each node, making use of a spring mechanics' analogy; see [11] for details.

The main extension of the ADMESH+ methodology for this work is to allow 


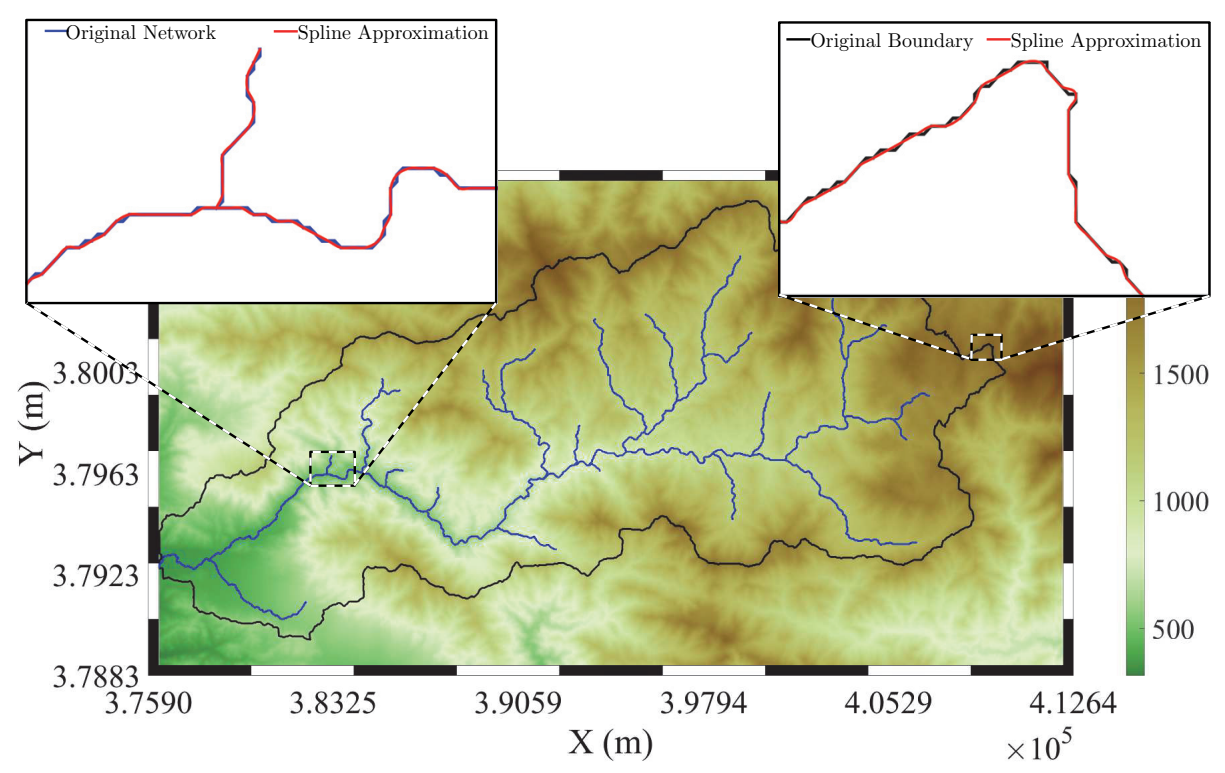

Figure 4: Insets showing the original representations of the channel network (left, blue line) and watershed boundary (right, black line) obtained from the DEM and the smooth spline approximations (red lines) used in ADMEsh+.

for interior constraints when generating the mesh. That is, we wish to compute a triangulation in which element edges are constrained along the specified channel network and watershed boundaries. Given that DEM data is typically recorded on a structured grid, the extracted channel networks and watershed boundaries will often exhibit a stair-case like appearance on the scale of the original DEM. Rather than constraining element edges to conform exactly to this representation, which can lead to low-quality elements on a scale smaller than desired in the neighborhood of the constraints, we compute and work with a smooth (Hobby) spline approximation [28] of the constraints, as illustrated in Figure 4.

The spline approximation allows for a simple calculation of the "curvature" of the constraints, which can then be taken into account in sizing elements along them. Specifically, the user can specify the number of elements desired 
per radian of curvature, which acts to place smaller element edge lengths in areas of high curvature, in order to capture the relevant geometry, but allows larger element edges to be used in straighter segments. A specific (uniform) element edge size along the constraints can also be specified by the user. Given these specified constraints, the ADMESH+ methodology described above was modified to first create a constrained Delaunay triangulation and then perform the force equilibrium calculations, where all nodes along the constraints are held fixed. Figure 5 shows an example of a constrained triangulation created by ADMESH+. The mesh contains 34,411 elements that range in size from approximately 50 to 400 meters and was generated automatically in 2.46 minutes. The element quality of the mesh, on a $0-1$ scale, where 0 is a completely degenerate triangle and 1 is an equilateral triangle, averaged 0.98 with a minimum element quality of 0.70 ; see [11] for more details on the mesh-quality measure.

In addition to accommodating interior constraints, ADMESH+ was also further developed to provide the underlying mesh data structure required by the described numerical approach. A "standard" mesh data structure consists of a list of nodal point coordinates, each associated with a unique identifier (ID), and an element connectivity list that contains the nodal point IDs defining a given element. This type of mesh data structure is part of the standard output of ADMESH+ (and all mesh generators). For the numerical approach considered here, the data structure needs to be enriched to include a list identifying all converging edges of the mesh, i.e., those edges whose attached elements both have topographic gradients (slope vectors $\mathbf{S}_{0}$ ) pointing towards the edge; see sketch of Figure 6. As flow propagates only in the direction of the slope vector for the kinematic wave approximation (that is, downhill), this list serves as the indication that flow into these edges will not be allowed to propagate through into the neighboring elements. Instead, the flow into the converging edges will act as lateral inflow, $\mathbf{q}_{L}$, into a one-dimensional kinematic wave approximation that is solved along that edge.

All edges along the original channel network will be converging edges. In addition to these, the (interpolated) topography of the triangulation may also 


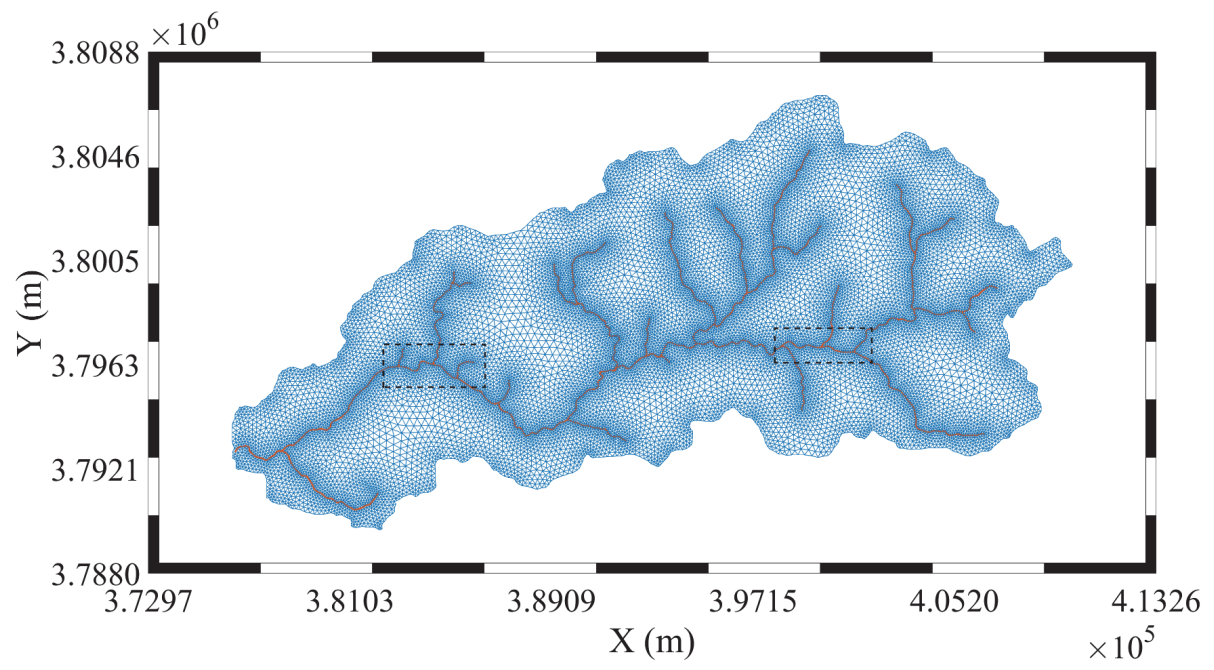

Figure 5: Example of a finite element mesh generated automatically by ADMESH+, where the red lines indicate the constrained channel network.

produce converging edges that are not part of the defined channel network (see red lines of Figure 6). For these types of converging edges, the collected overland flow is routed to a node on the defined channel network via the steepest path. A one-dimensional kinematic wave approximation is solved over all edges along this path; Figure 6 illustrates an example of these concepts, where all converging edges and flow paths are highlighted. 


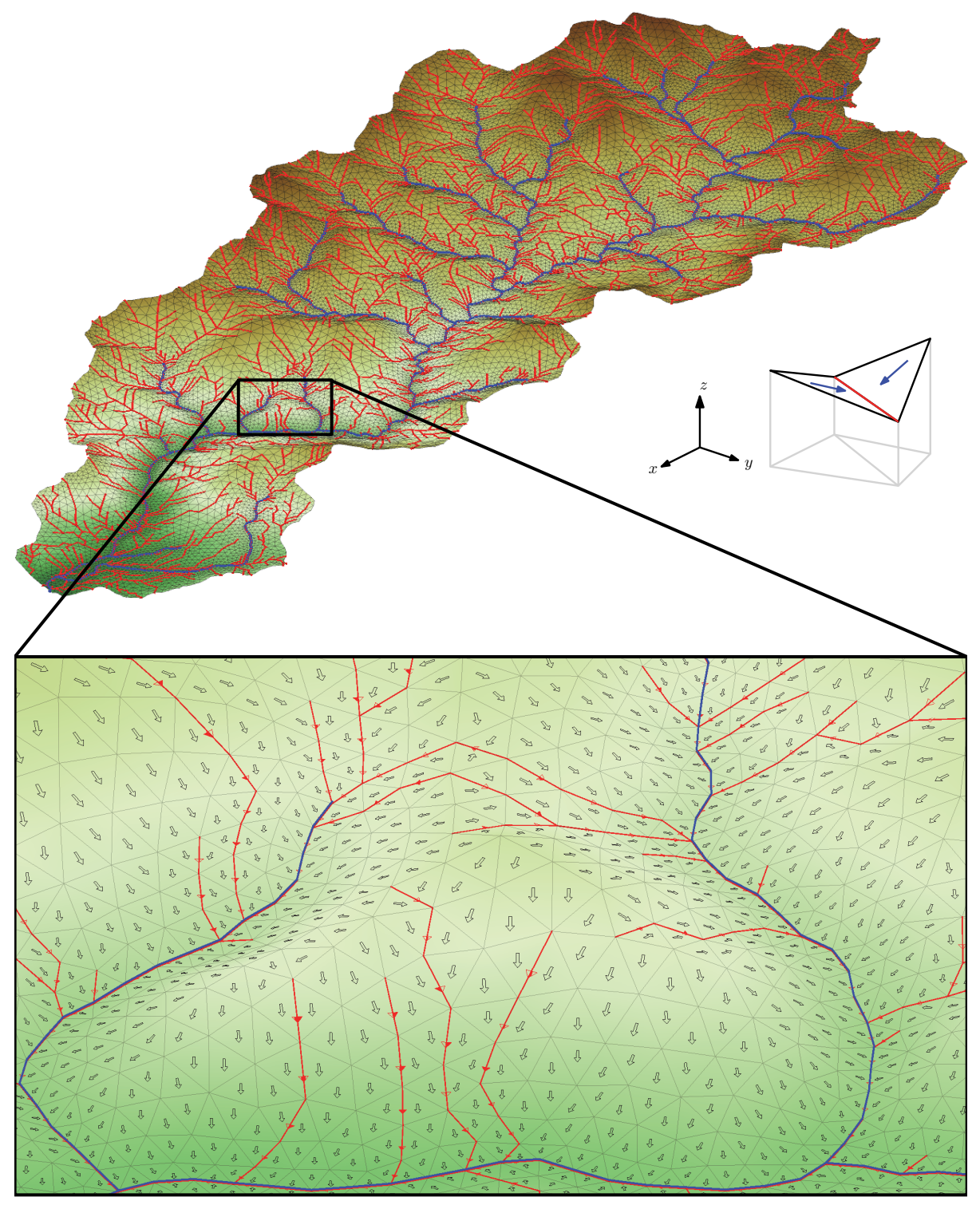

Figure 6: A three-dimensional profile view of a finite element mesh produced by ADMESH+ for a sample watershed, where blue lines indicate edges that are part of the original constrained channel network, red lines indicate one-dimensional overland flow routing edges and large open arrows indicate flow direction over each element face. The sketch to the right illustrates a converging edge. 


\section{Numerical Results}

In this section, the developed model is applied to four test cases, comparing the numerical results to both analytic solutions and experimental data. In what follows, the developed model is referred to as the DG-SAKE (= SectionAveraged Kinematic wave Equation) model. In the first two test cases, we verify the DG-SAKE model against known analytic solutions for the kinematic wave approximation equations for both overland and open-channel flow. This is followed by model validation against two sets of experimental results that validate the overland and open-channel flow components of the model. Both the model verification and validation studies examine first the overland flow component of the model system, followed by the testing of the fully coupled overland flow/open-channel multidimensional approach used by the model

\subsection{Overland flow on an impervious plane}

In $[29,30,31]$, Singh uses the method of characteristics to derive analytic solutions to the one-dimensional kinematic wave equation for flow occurring over a plane due to rainfall under several different scenarios, such as storms moving up or down an impervious or infiltrating plane. Here, we examine the basic case of overland flow occurring from uniform rainfall over an impervious plane (see [32] for an application of the DG-SAKE model to the moving storm test case of Singh and details on the analytic solutions). This is a commonly used verification test case for overland flow models; see, for example, [33, 34, 35]

A schematic of the problem is shown in Figure 7, where the specific numerical values that are used here are indicated. An initial condition of $H(x, 0)=0$ is taken over the entirety of the domain and an upstream boundary condition of $H(0, t)=0$ is specified for all time $t$. The uniform rainfall rate of $r_{\circ}=50.8$ $\mathrm{mm} / \mathrm{h}$ is applied for a duration of $T=30$ minutes at which point the rain stops.

The problem is solved over a one-dimensional mesh using a uniform element size of $9.25 \mathrm{~m}$ (20 elements) and a time step of $\Delta t=1.5 \mathrm{~s}$. A simulation time of 1 hour is used, and piecewise polynomial spaces of degree $p=0$ and $p=1$ 


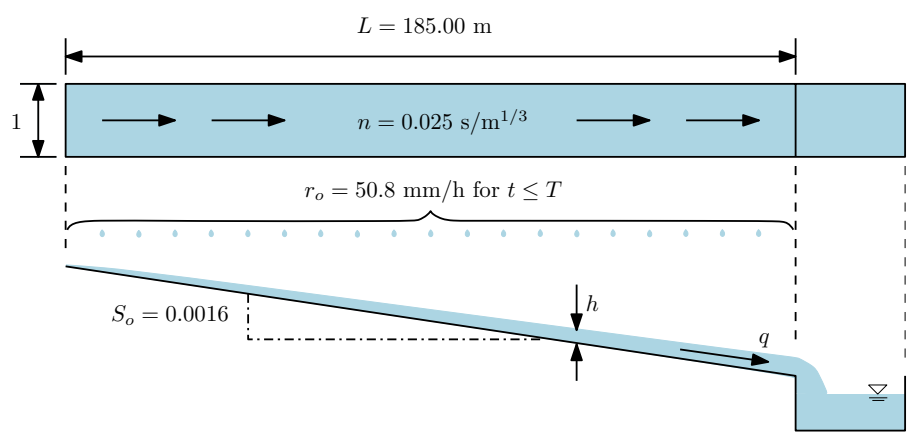

Figure 7: A schematic of the impervious plane test case subjected to a uniform rainfall rate of duration $T$.

are employed. The computed and analytic outflow discharges as a function of time (that is, the outflow discharge hydrographs) are shown in Figure 8. The numerical solutions compare well with the analytic solution; however, as shown in detail in the zoom-in of Figure 8 , the $p=0$ solution fails to adequately capture the latter part of the rising limb and the peak discharge at this mesh resolution. Note, however, that the $p=1$ solution at the same mesh resolution provides a much more accurate solution, sharply resolving the rising limb leading up to the peak discharge. Figure 9 , shows a second solution using a $p=0$ approximation, this time using 640 elements. At this mesh resolution, the $p=0$ approximation provides a solution comparable to the 20-element, $p=1$ solution but at the expense of using 32 times the mesh resolution. More specifically, the 20-element, $p=1$ solution has a root-mean-square error at the outflow point (over the duration of the simulation) of approximately $2.2 \times 10^{-11}$, which is two orders of magnitude lower than the 20 -element, $p=0$ solution error $\left(\approx 3.9 \times 10^{-9}\right)$. This result suggests the potential benefits of using $p$ (polynomial) refinement versus standard $h$ (mesh) refinement for modeling overland flow.

Figures 8 and 9 can also be compared to Figure 4 of [34], which compares the analytic solution of this test case to numerical results from two different models using a normalized mesh spacing $(\Delta x / L)$ of 0.0025 . The DG-SAKE model is providing visually comparable results to these models at a much coarser (by an order of magnitude) normalized mesh spacing of 0.05 . 

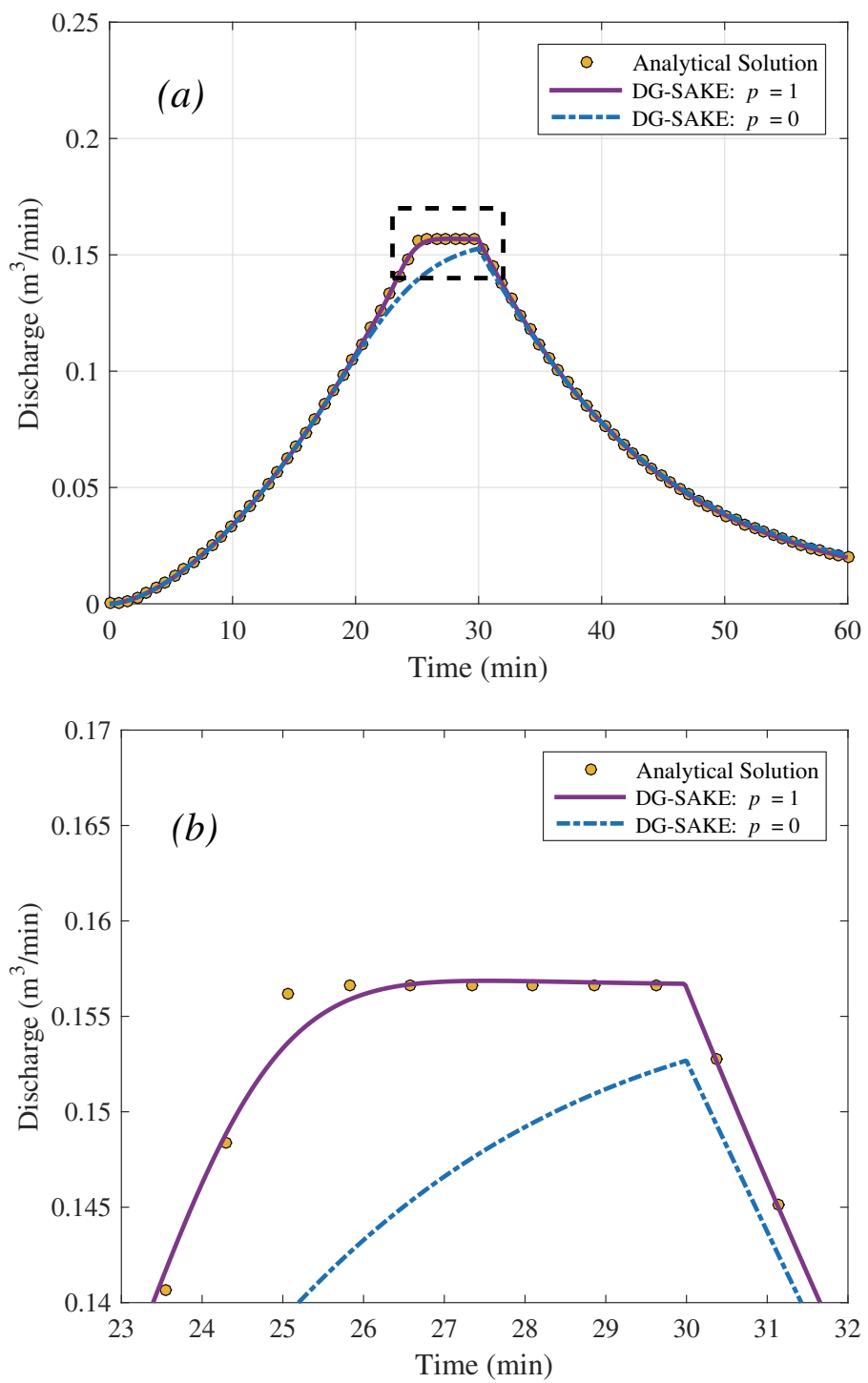

Figure 8: Outflow discharge hydrographs of the impervious plane test case for a uniform rainfall rate of duration $T=30$ minutes, showing the analytic solution (filled yellow circles), along with the DG-SAKE $p=0$ (dashed blue line) and $p=1$ (solid purple line) solutions using 20 elements, for (a) the entire simulation period and (b) a zoom-in at peak discharge, where it can be observed that the $p=0$ solution fails to adequately capture the peak discharge at the current mesh resolution. 

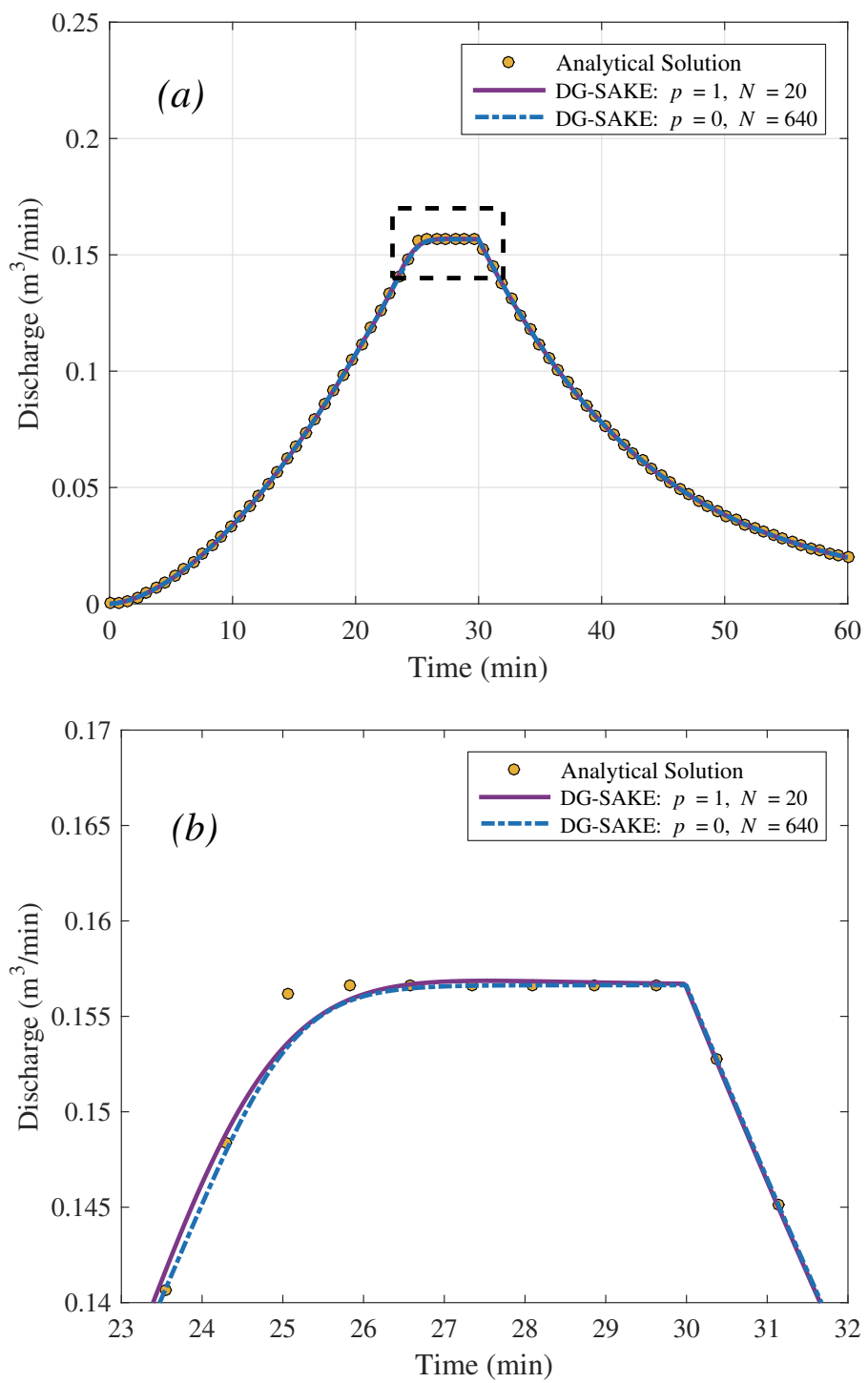

Figure 9: Outflow discharge hydrographs of the impervious plane test case for a uniform rainfall rate of duration $T=30$ minutes, showing the analytic solution (filled yellow circles), along with the DG-SAKE $p=0$ (dashed blue line) and $p=1$ (solid purple line) solutions, using $N=640$ and $N=20$ elements, respectively, for (a) the entire simulation period and (b) a zoom-in at peak discharge, where it can be observed that the $p=0$ and $p=1$ solutions are now comparable but at the expense of the $p=0$ solution using 32 times the mesh resolution. 


\subsection{The catchment-stream problem}

In order to test both the overland and open-channel flow components of the model, as well as the coupling mechanism between them, we examine the catchment-stream problem proposed by Wooding [36], which provides analytic solutions for both the overland and open-channel flow resulting from a uniform rainfall rate $r$. A schematic of the problem is shown in Figure 10, which consists of two identical rectangular planes joined to form a $\mathrm{V}$. The line of intersection of the planes (the width of the channel shown in Figure 10 is for illustrative purposes) is taken to be a stream (an open channel) with a slope of $S_{0}$ in the $y$ direction . (Note: This slope is neglected on the catchment surfaces in both the analytic solution provided by Wooding and the mesh representation used here as shown in Figure 11.)

The numerical values we use for this test case are shown in Figure 10. Specifically, the (impervious) overland flow planes are taken to be of dimension 1000 $\mathrm{m} \times 800 \mathrm{~m}$, with a constant downward slope in the $x$ direction of $S_{0_{x}}=0.05$ and, as noted above, no slope in the $y$ direction (denoted by $S_{0_{y}}$ in the figure). The width of the channel at the intersection of the planes is taken to be 20 m. The overland flow planes are subjected to a uniform rainfall rate of 10.8 $\mathrm{mm} / \mathrm{h}$ for a duration of 1.5 hours, and the Manning's roughness coefficients in the overland and open-channel flow equations are set to $n=0.015$ and $n=0.15$, respectively. As in the previous test case, both the initial water depths and the upstream flow boundary conditions are taken to be zero. The problem domain is discretized using the triangular mesh shown in Figure 11, which consists of 120 two-dimensional overland flow elements and 6 one-dimensional channel elements of equal length, formed by the edges of the triangular elements along the line $x=800$.

The problem is solved with the DG-SAKE model using piecewise polynomial spaces of degree $p=2$ for both the overland and open-channel flow DG discretizations. A duration of 3.3 hours is simulated using a time step of $\Delta t=35.8$ seconds. The outflow discharge hydrograph for one of the overland flow planes at a point along the bottom of the plane (i.e., along the line $x=800$ ) is shown 
472 in the top plot of Figure 12, where it can be observed that good agreement is obtained between the analytic and DG-SAKE solutions. As described in section 3.2.1, the computed discharges along the edges of the triangular elements at the bottom of the planes are fed into the one-dimensional component of the model as lateral inflow source terms $\left(q_{L}\right.$ of Equation 3$)$. The resulting outflow discharge hydrograph for the open-channel at point $(x, y)=(800,0)$ is shown in the bottom plot of Figure 12, where again good agreement between the model results and the analytic solution can be observed - in this case, up to the point of the recession limb of the hydrograph. (Note: Evaluation of the analytic solution for the recession limb portion of the stream outflow hydrograph proved problematic due to its complexity, see equations on page 264 of [36].) As with the previous test case, the DG-SAKE model results can also be compared to Figure 4 of [34], which visually compares results from several different models for this problem. Again, the DG-SAKE model is producing (visually) comparable result at a much coarser mesh resolution (compare the meshes of Figure 11 of this work and Figure 3 of [34]). 


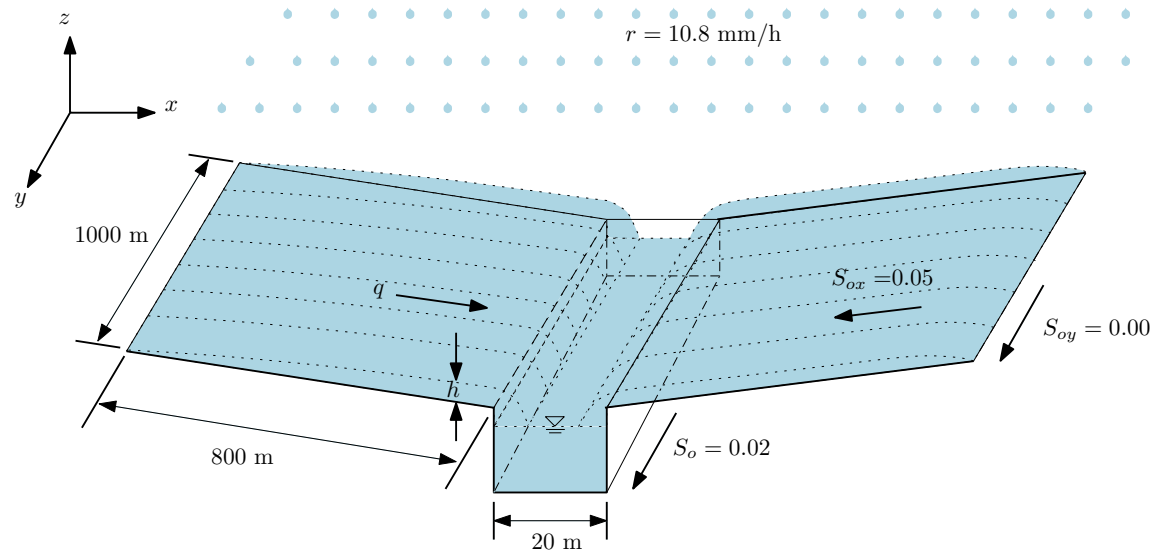

Figure 10: A schematic of the V-shaped catchment problem of Wooding [36], showing the specific numerical values used here.

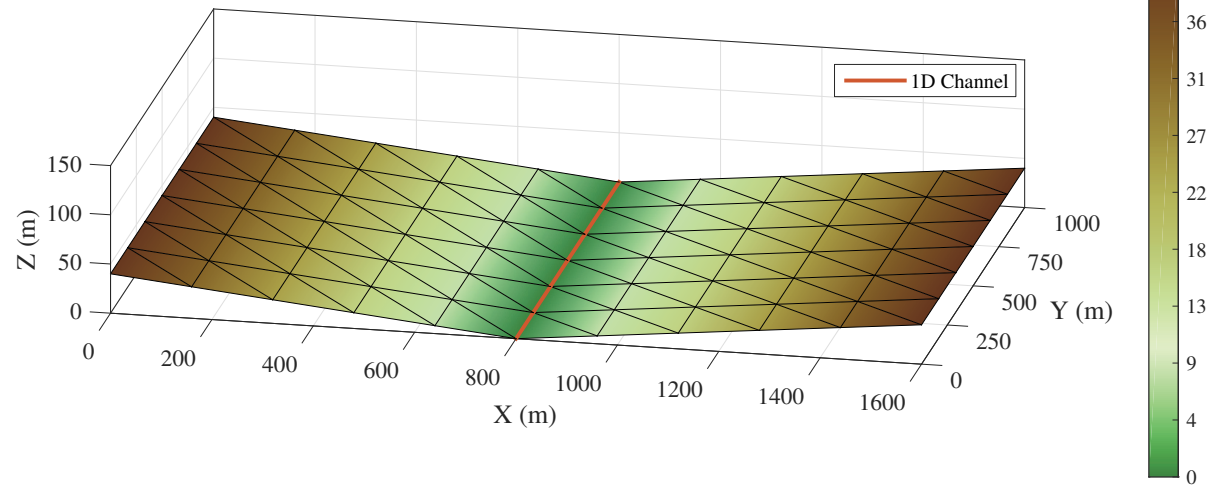

Figure 11: The finite element mesh used for the V-shaped catchment problem, which consists of 120 triangular overland flow elements and 6 one-dimensional (1D) channel elements of equal length (red line). Side color bar and colors on the mesh indicate elevation $z$. 

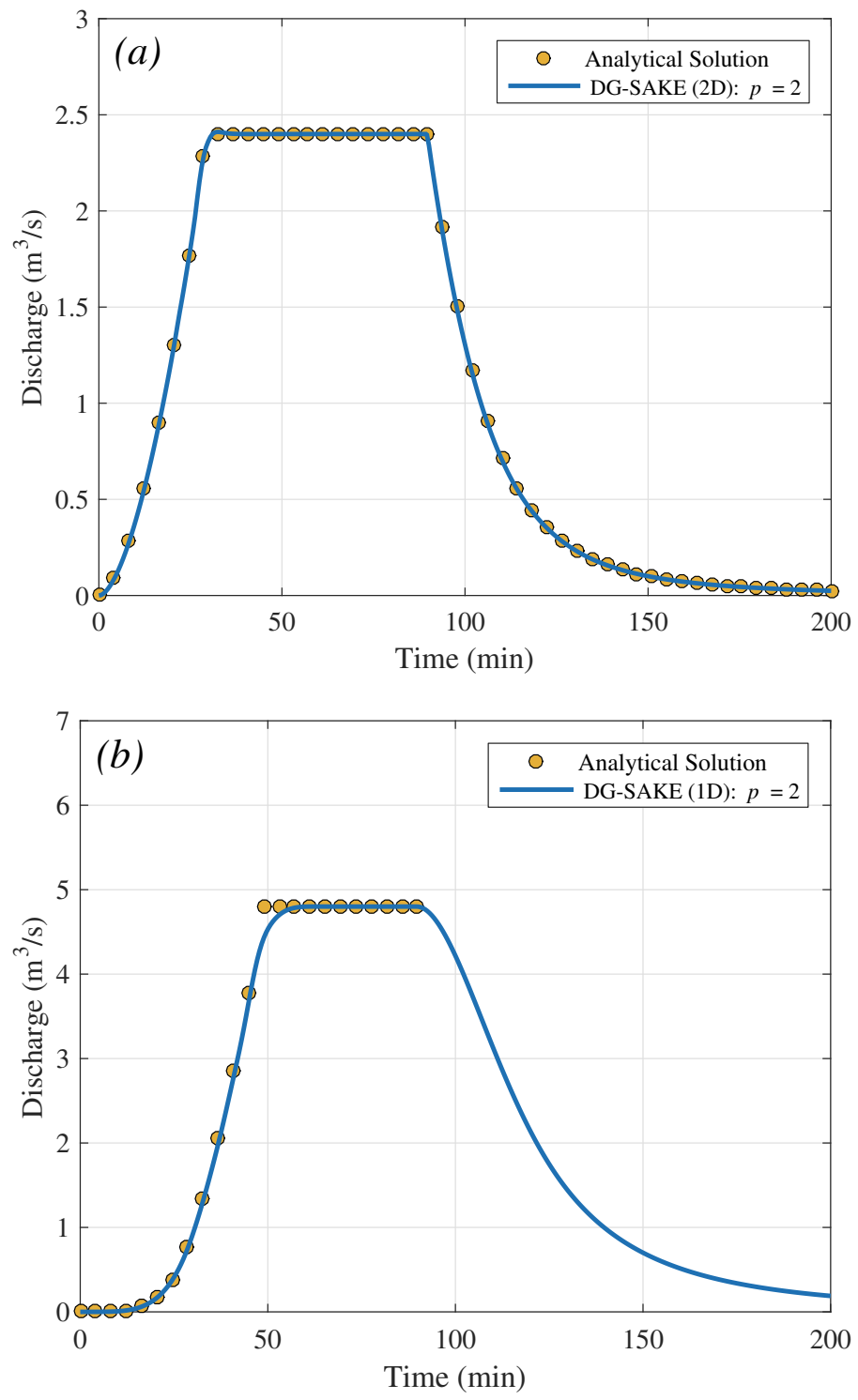

Figure 12: Outflow discharge hydrographs for (a) the two-dimensional (2D) overland flow at a point along $x=800$ (from one of the planes) and (b) the one-dimensional (1D) open-channel flow at point $(x, y)=(800,0)$, where it can be observed that the analytic solutions (filled yellow circles) and the DG-SAKE $p=2$ solutions (solid blue lines) are in good agreement. (See note in text regarding the analytic solution for the recession limb of the stream outflow discharge hydrograph). 


\subsection{Iwagaki's experiment}

Next, we validate the overland flow component of the DG-SAKE model against a set of well-known experimental results from Iwagaki [37], which have been used in a number of validation studies; see, for example, [38, 39, 40]. Figure 13 shows a schematic of Iwagaki's experimental setup, which consisted of an aluminum flume made up of three rectangular sections of progressively steeper slope in moving from top to bottom (the specific dimensions are shown in the schematic). These three planes were (simultaneously) subjected to rainfall intensities (uniform over a given plane) of rates $r=0.1080,0.0638$ and 0.0800 $\mathrm{cm} / \mathrm{s}$, respectively from top to bottom. Three different experiments were conducted with rainfall durations of $T=10,20$ and 30 seconds being applied over each plane for a given experiment.

We discretize the Iwagaki test domain using 24 rectangular elements, with each of the 3 planes consisting of eight elements of length of $0.196 \mathrm{~m}$ as shown in Figure 14. The Manning's roughness coefficient is set to $n=0.009$ (as reported by Iwagaki), and numerical simulations were run for a total of 70 seconds with a time step $\Delta t=0.10$ seconds. Simulations were performed for each of the three rainfall durations, $t_{d}=10,20$ and 30 seconds, using piecewise polynomial spaces of degree $p=1$. Numerical solutions for the outflow discharge hydrographs along with Iwagaki's experimental data at the outlet of the domain are shown in Figures $15 \mathrm{a}-15 \mathrm{c}$.

The results show that the DG-SAKE model performs very well for the short duration test case (plot (a) of Figure 15), with output matching the rising limb, peak discharge and recession limb of the hydrograph. For cases (b) and (c), the longer duration cases, the numerical solutions tend to predict the peak runoff of the discharge too early. Also, in case (b), the peak numerical discharge is slightly over estimated; however, the numerical results for cases (b) and (c) still provide reasonable approximations to the experimental data (without calibration), especially during the final phases of the recession limbs of the hydrographs. Additionally, it should be noted that the errors observed are most likely a result of the simplifying assumptions inherent in the kinematic wave approximation, 

i.e., the remaining dynamic terms of the momentum equation are not negligibly small as assumed (see Section 2.3). A number of studies have investigated the criteria for which the kinematic wave approximation is suitable; see, for exam522 ple, $[12,13,41]$ (in particular see the "contour" maps of [12]). These criteria 523 involve a dimensionless parameter termed the kinematic wave number $K$. Withnumbers associated with cases (b) and (c) tended to (low) values of $K$ for which the kinematic wave approximation is not as valid; see [12]. In these situations, the $K$ value of the numerical solution could actually be used to monitor the applicability of the kinematic wave approximation and act at a threshold value for switching from the kinematic wave approximation to the full shallow water equations. This idea is discussed in more detail in Section 5.4.2. 


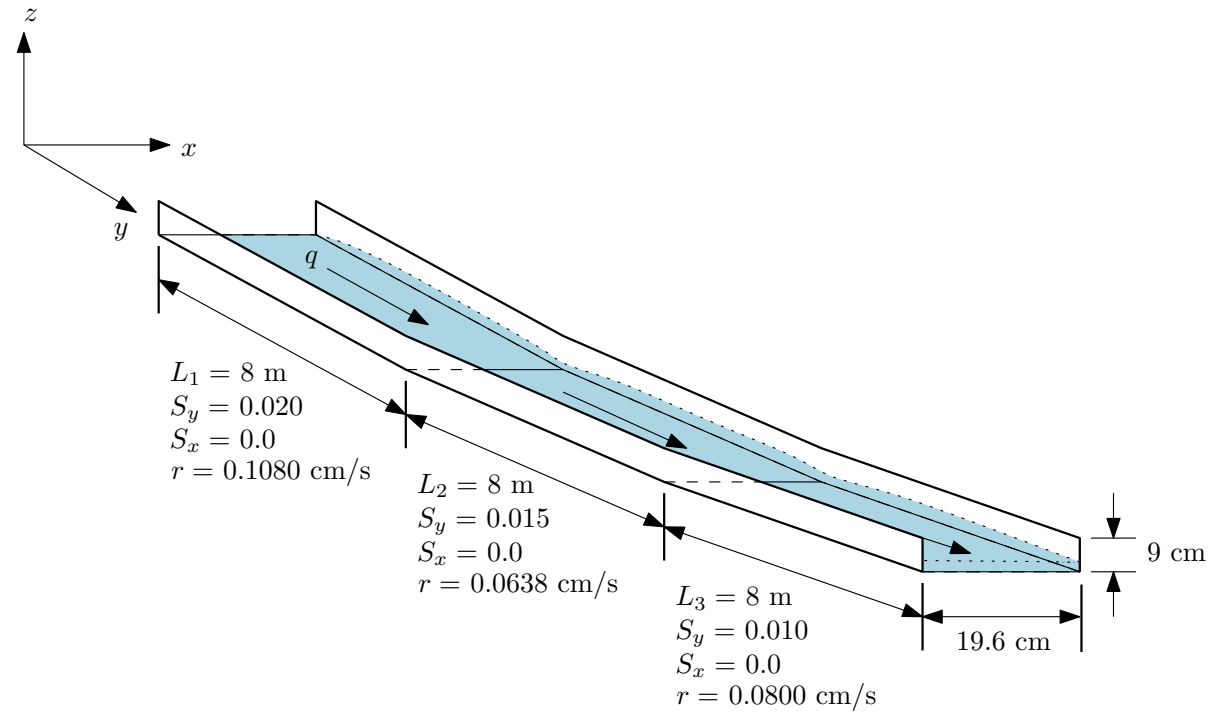

Figure 13: A schematic of the experimental flume used in Iwagaki's Experiment.

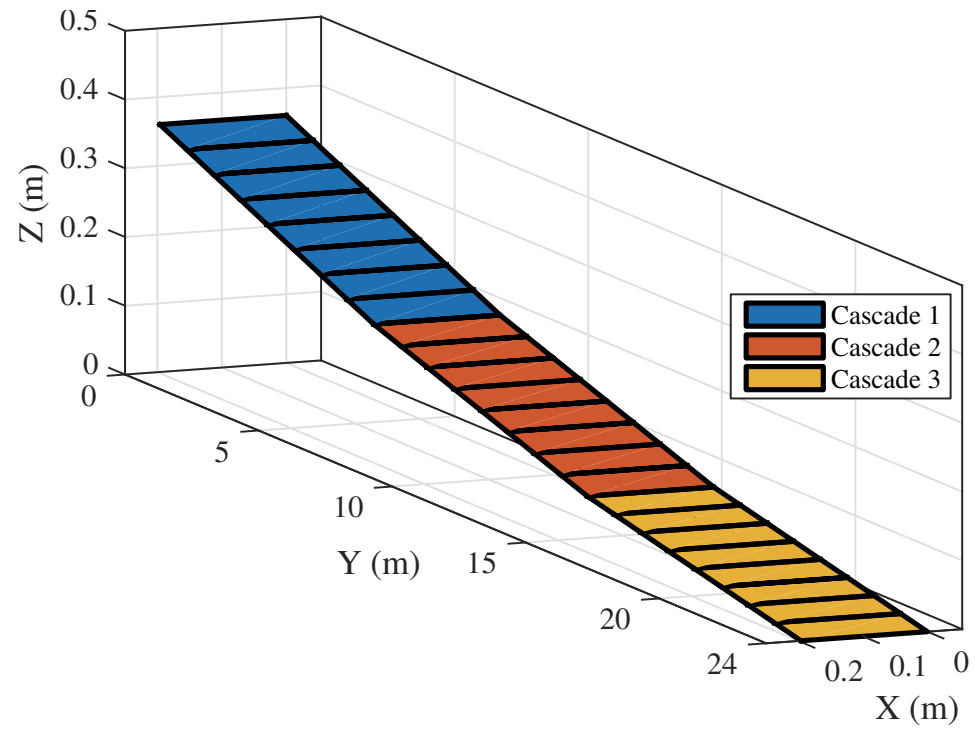

Figure 14: Finite element mesh of Iwagaki's experimental flume. 

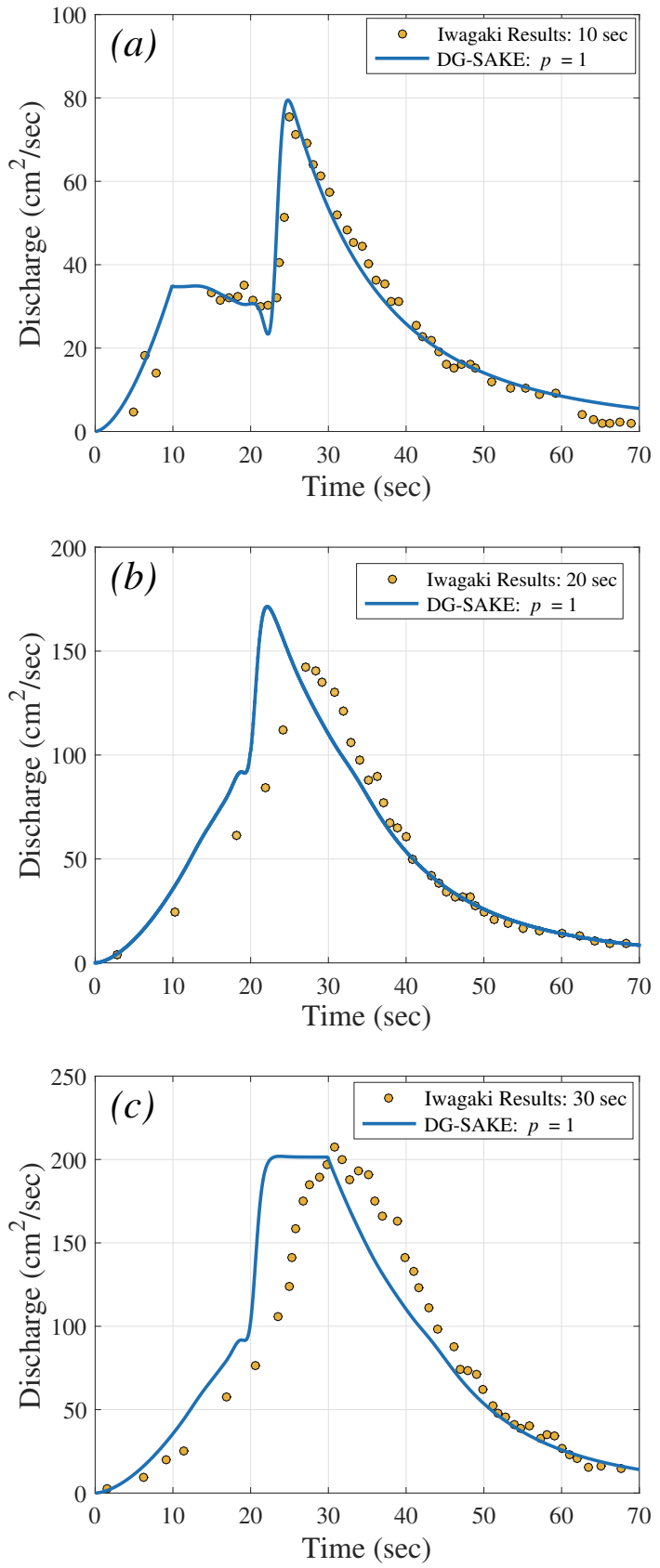

Figure 15: Outflow discharge hydrographs for the Iwagaki test case, comparing the experimental results (filled yellow circles) and the DG-SAKE $p=1$ solutions (solid blue lines), for the experiments of duration (a) $T=10$, (b) $T=20$ and (c) $T=30$ seconds. 


\subsection{Goodwin Creek experimental watershed}

In this final test case, results from the coupled overland and open-channel flow components of the DG-SAKE model are validated against data from the Goodwin Creek Experimental Watershed (GCEW) established by the U.S. Department of Agriculture's National Sedimentation Laboratory in 1981 [42]. The GCEW, located in Panola County, Mississippi, is extensively monitored and has served as a platform for several hydrology and model validation studies; see, for example, $[43,35,42,44,45,46]$

Numerical results from the DG-SAKE model (discussed in detail below) are compared to observational data from on-site stream-flow gauges and to numerical results from a calibration study performed with the CASC2D model - a well-established, finite difference-based model for simulating overland and open-channel flow; see $[44,45,47]$. For consistency in comparison, input data used by DG-SAKE (with the exception of the computational mesh) was the same as that used by the CASC2D model (obtained online from [44]). This includes elevation, land-cover, soil-type and precipitation data as discussed in the next subsection. The infiltration term of Equation (4) was computed using the Green-Ampt model in DG-SAKE; see [32] for more details. No calibration of the DG-SAKE model was performed.

\subsubsection{Model input data}

Elevation (DEM), soil-type and land-cover data are provided on $90-\mathrm{m} \times 90$ $\mathrm{m}$ spaced grids as shown in the plots of Figure 16. The horizontal extent of the GCEW DEM is approximately $21.3 \mathrm{~km}^{2}$ with a relief of about $57 \mathrm{~m}$. This DEM serves as the input to construct the finite element mesh used by DGSAKE as described below. With regard to the soil-type and land-cover data, seven different soil types and four different land-cover classifications have been identified in the GCEW; see middle and bottom plots, respectively, of Figure 16. This data informs the values of the Green-Ampt infiltration model parameters and the Manning's roughness coefficients used in DG-SAKE for the overland flow model component. (Note: a uniform Manning's roughness coefficient of 
$n=0.035$ was used in the open-channel flow component of the model and cross sections were approximated as being rectangular). Additional information on this data can be found in the extensive documentation provided in [42, 45].

From the GCEW DEM, the channel network is first identified and then approximated, along with the boundary of the domain, by a smooth spline using the approach described in Section 3.3; see Figure 17. Given these approximations, a finite element mesh is then generated using ADMESH+, insuring that element edges are aligned along the defined channel network as shown in Figure 18. (Note: elevation data at element nodes and at points within an element required for computation, i.e., at numerical integration points, is obtained from a bilinear interpolant constructed from the original DEM data). As demonstrated in the example of Section 3.3, converging edges that are not part of the defined channel network can occur and are identified in the mesh data structure provided by ADMESH+; see red lines of Figure 19. The constructed mesh consists of nearly 4,000 elements, ranging in size from approximately 58 to 187 m, exhibits an average mesh quality of 0.97 (see discussion of Section 3.3) and was generated automatically in 20 seconds.

Finally, input data for precipitation was constructed from data obtained from sixteen precipitation gauges located within (or just outside) the GCEW (see Figure 19). The particular storm event used had a rainfall duration of 4.8 hours, an average rainfall depth of $73.6 \mathrm{~mm}$ and exhibited average and maximum rainfall rates of 14.7 and $51.6 \mathrm{~mm} / \mathrm{h}$, respectively, with very little rainfall preceding the event [45]. Rainfall rates used in DG-SAKE were interpolated spatially at all computational points using an inverse distance (squared) weighting and interpolated linearly in time. 

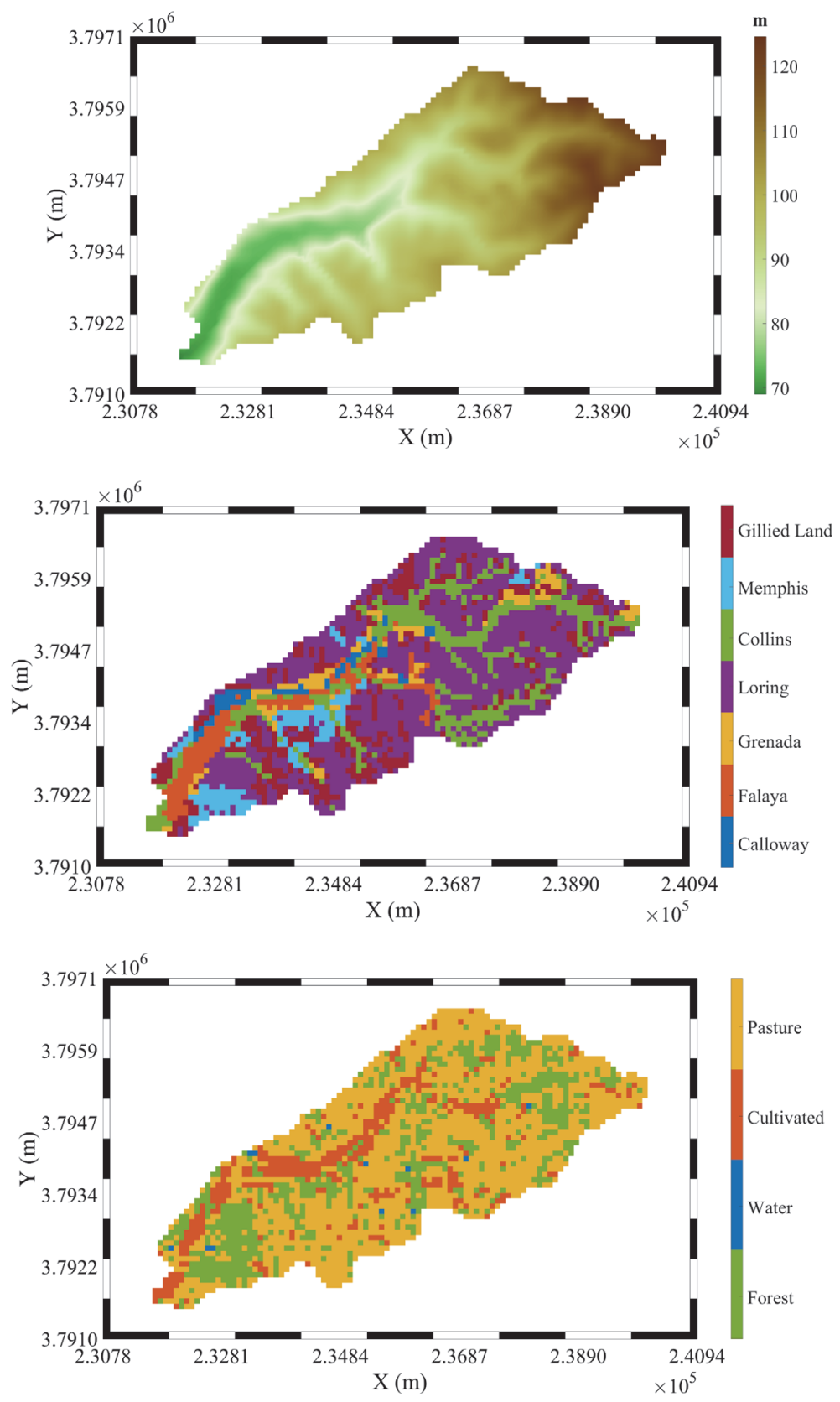

Figure 16: Contour maps of the 90-meter-spaced elevation (top), soil-type (middle) and landcover (bottom) data for the GCEW test case as used by DG-SAKE and the CASC2D model. 


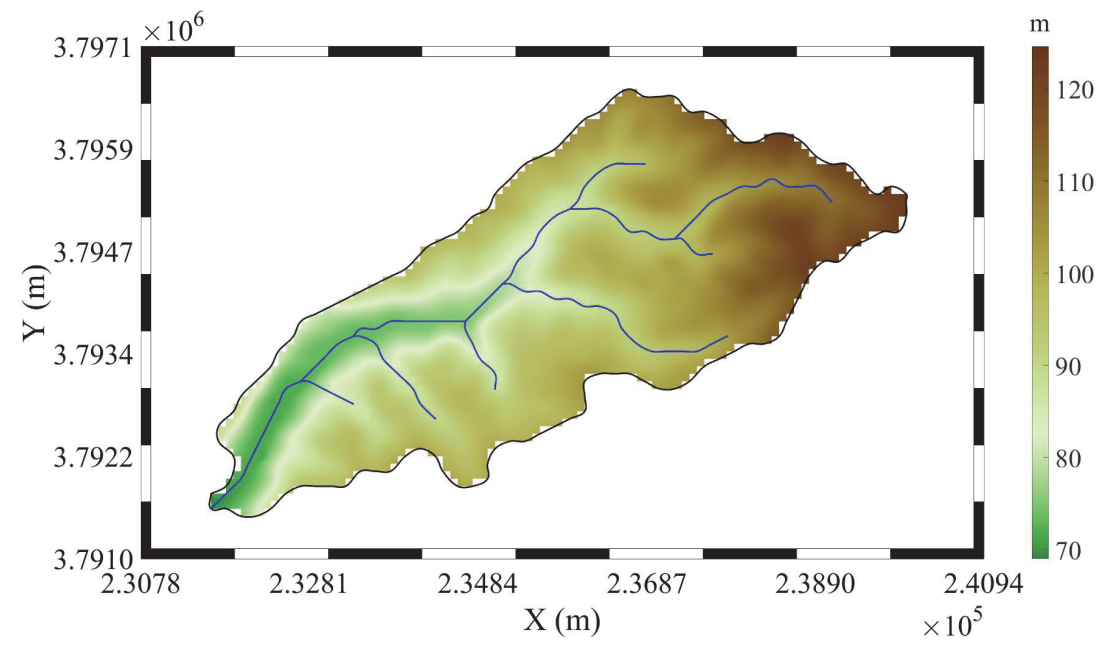

Figure 17: The smooth spline approximation of the the channel network (blue curves) and the boundary (black curve) obtained from the GCEW DEM (colors indicating elevation).

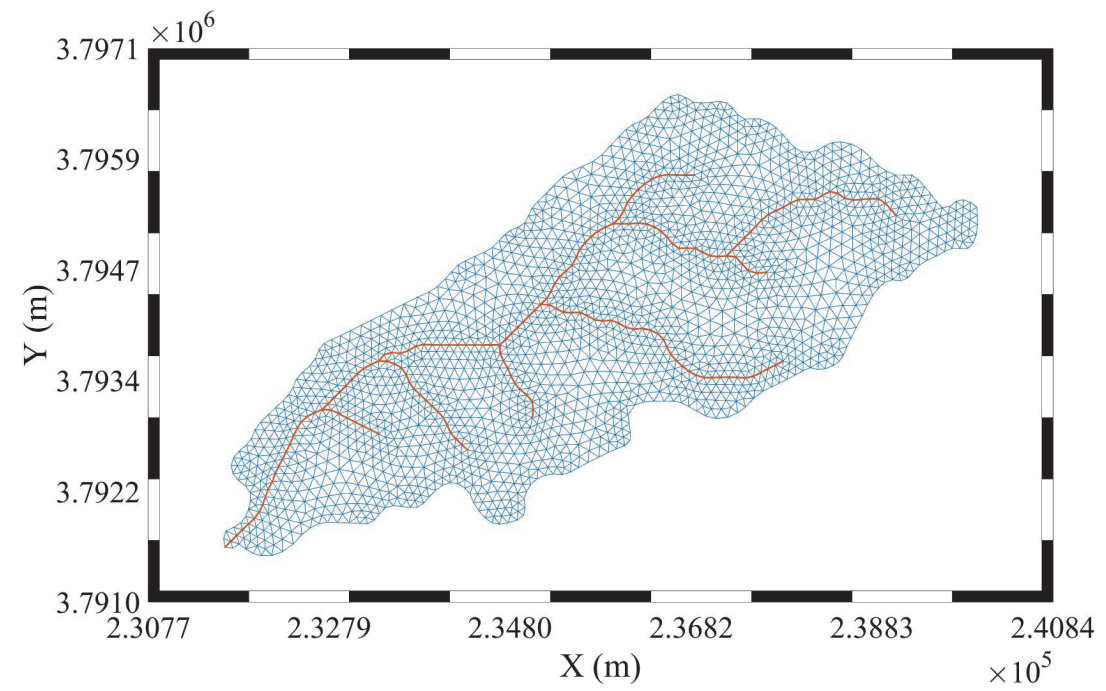

Figure 18: Finite element mesh of the GCEW domain generated using ADMESH+, with the preserved channel network shown in red. 


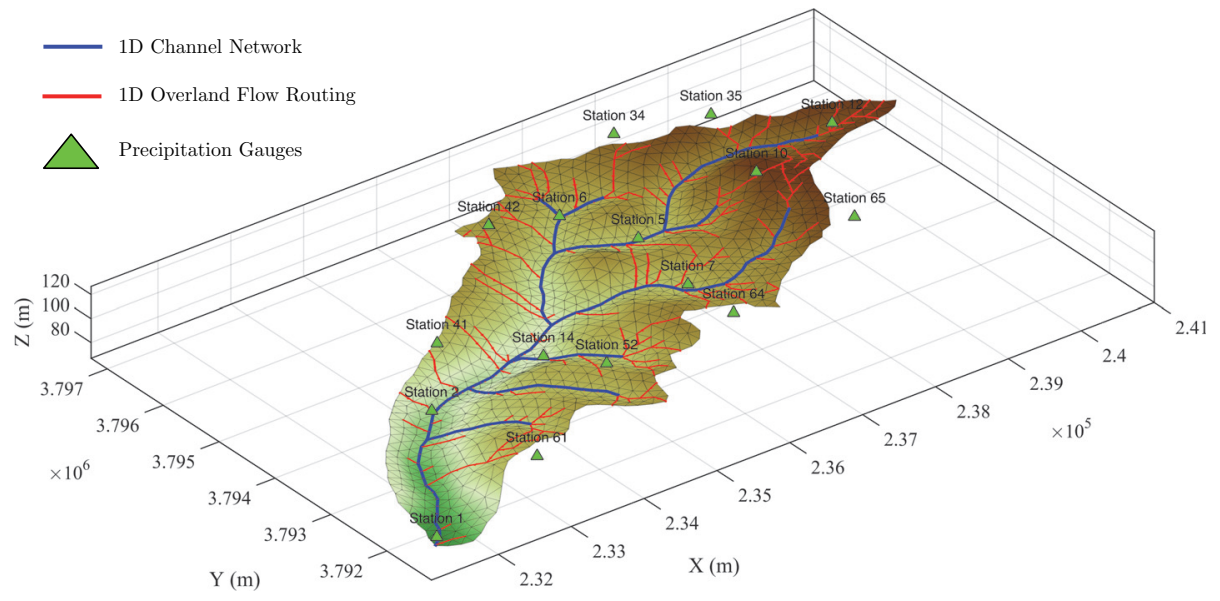

Figure 19: Location of the precipitation gauges (green triangles) used in the GCEW test case shown on the finite element mesh, where the blue and red lines indicate the $1 \mathrm{D}$ channel network and overland flow routing paths, respectively.

\subsubsection{Model results and comparison}

Two numerical simulations were performed for this test case using DGSAKE. The first simulation uses piecewise constant, $p=0$, DG spatial approximations, while the second uses higher-order piecewise quadratic, $p=2$, approximations. A duration of 13 hours was simulated in each case, with different time steps being used for the $p=0$ and $p=2$ cases based on the CFL condition ( $\Delta t=42$ and 32.02 seconds, respectively). Results from both simulations are compared to observational data and CASC2D model results located at six stream discharge gauges distributed throughout the GCEW (numbered 1, 4, 6, 7, 8 and 10 as shown in Figure 20). Hydrograph results shown in Figures 21 and 22 (for $p=0$ and $p=2$, respectively) are presented in units of stream velocity $V(\mathrm{~mm} / \mathrm{h})$, which is computed by dividing the discharge at each station by the sub-basin area contributing flow to that station. Table 1 provides a more quantitative assessment of the results, the specific contents of which are discussed in detail below. The DG-SAKE simulations were performed on a 64bit DELL Precision M4800 machine with 16-GB RAM and a 2.40-GHz Intel i7 


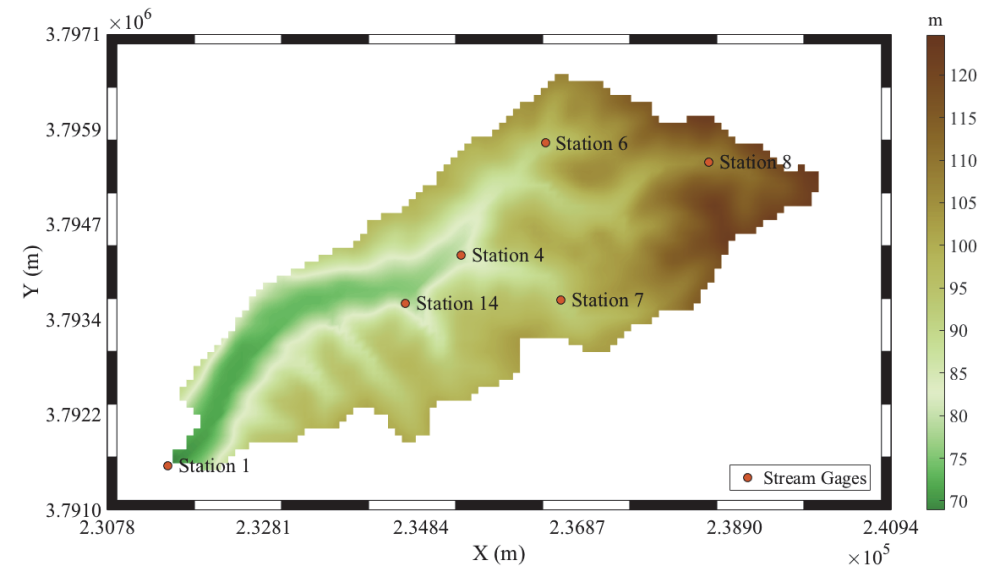

Figure 20: Location of stream gauges (filled orange circles) in the GCEW used in the validation study.

processor. The CPU time for the first simulation, which has 3,944 total degrees of freedom, was 14.47 seconds, and the CPU time for the second, which has 23,664 degrees of freedom, was 6.54 minutes.

First, as can be observed in the plots of Figure 21, the $p=0$ DG-SAKE simulation results are, in general, comparable to those of the CASC2D model. Both models accurately predict the time to peak flow and capture the general temporal character of the rising and recession limbs of the hydrographs (with the CASC2D model generally providing slightly more accurate representations of the recession limbs). One exception to the second observation is the noticeable lag time in the DG-SAKE simulation of the rising limb at station 1. We comment on this at greater length below. Magnitudes of the peak flow are captured with varying degrees of accuracy at the six gauge locations, with more sizable errors in both models being observed at gauge station numbers 6,8 and 14. (It is noted in [45] that these gauges correspond to the smallest sub-basins.)

As was observed in the previous test cases, one (numerical) strategy for improving model results is the use of $p$ refinement. Indeed, the $p=2$ DGSAKE results provide notably more accurate predictions of the magnitudes of the peak flow as can be observed in the plots of Figure 22 (while continuing to accurately capture the time to peak). Table 1 provides a quantitative measure 
${ }_{621}$ of these improvements, where the percent decrease in error achieved with $p$ ${ }_{622}$ refinement (i.e., in going from $p=0$ to $p=2$ ) is shown. With all but the first ${ }_{623}$ gauge station, significant decreases in error are observed, ranging from 24 to 91 ${ }_{624}$ percent. The average (over all gauge stations) of the absolute errors in peak flow ${ }_{625}$ for the CASC2D and DG-SAKE model simulations are also shown in Table 1, ${ }_{626}$ where it can be observed that the $p=2$ DG-SAKE solution provides the lowest ${ }_{627}$ average error and the lowest error at four of the six gauge stations (numbers in ${ }_{628}$ bold in the table). 

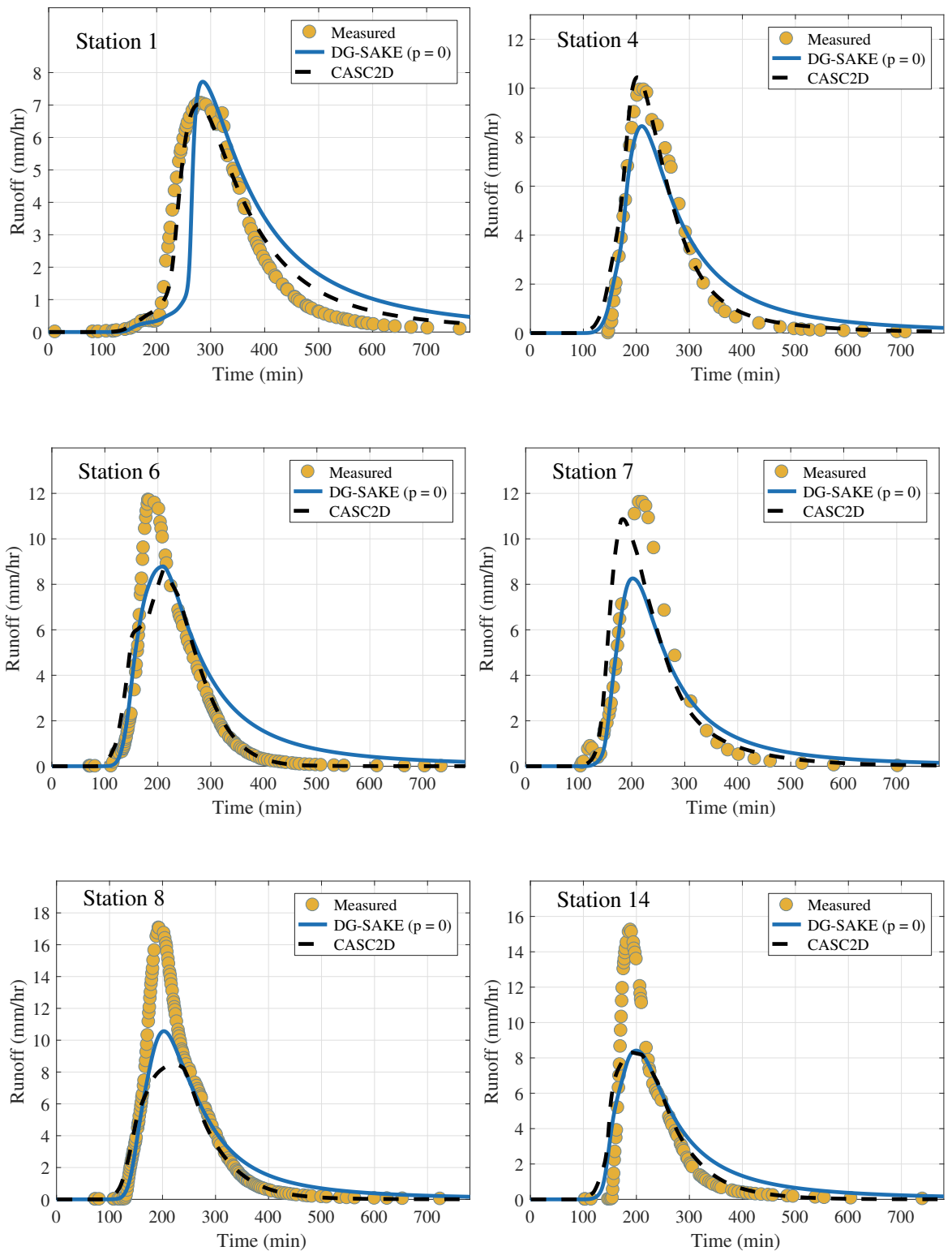

Figure 21: Hydrograph results at six stream gauges in the GCEW, comparing observational (measured) data (filled yellow circles), the CASC2D model results (dashed black lines) and the DG-SAKE $p=0$ model results (solid blue lines). 

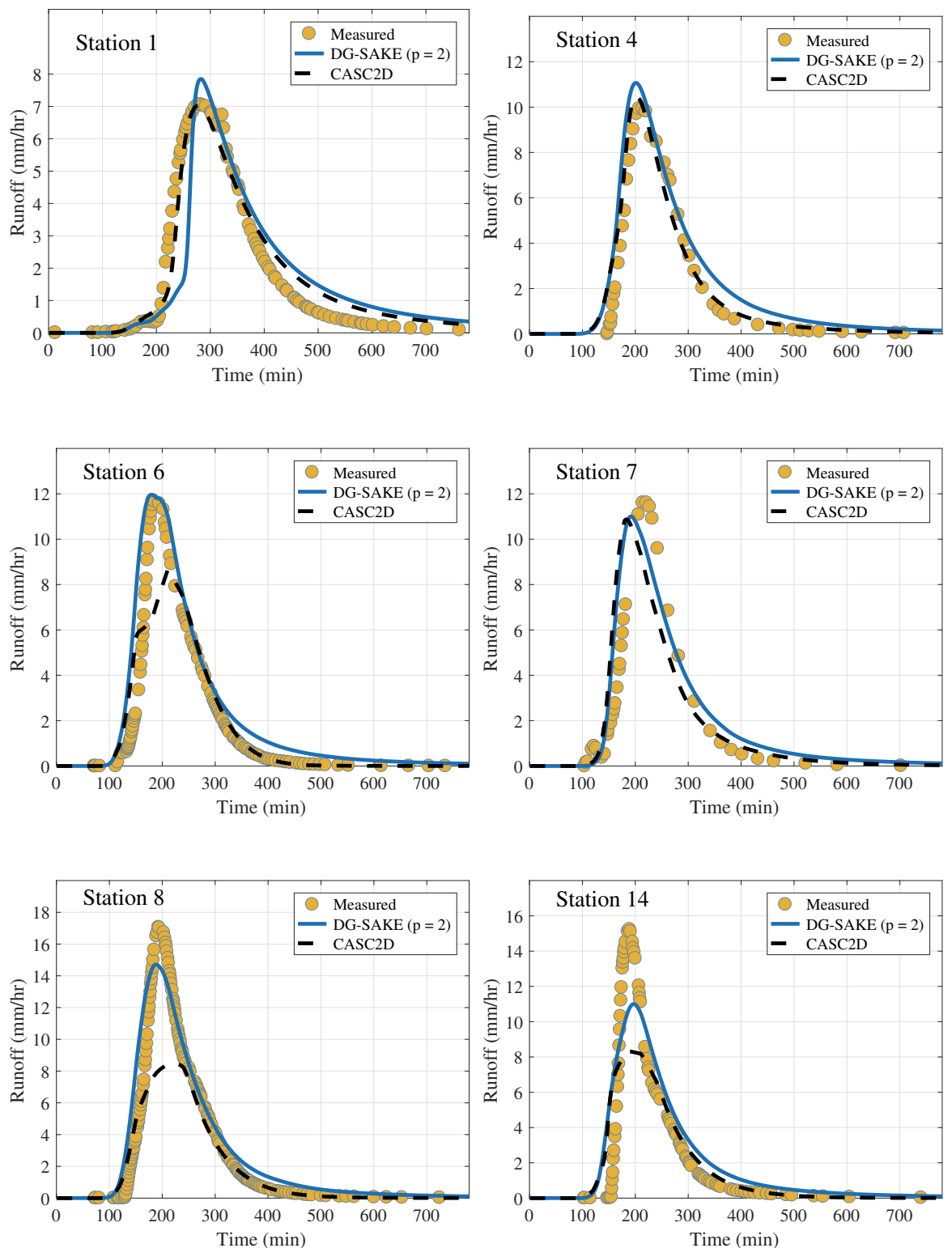

Figure 22: Hydrograph results at six stream gauges in the GCEW, comparing observational (measured) data (filled yellow circles), the CASC2D model results (dashed black lines) and the DG-SAKE $p=2$ model results (solid blue lines). 
Table 1: Peak discharge summary of model results.

\begin{tabular}{|c|c|c|c|c|c|c|c|c|}
\hline \multirow{3}{*}{ Gauge } & \multicolumn{4}{|c|}{$\begin{array}{c}\text { Peak flow rate, } V \\
(\mathrm{~mm} / \mathrm{hr})\end{array}$} & \multicolumn{3}{|c|}{$\begin{array}{l}\text { Absolute error } \\
\qquad(\mathrm{mm} / \mathrm{hr})\end{array}$} & \multirow{3}{*}{$\begin{array}{c}\% \text { Decrease } \\
\text { in error } \\
\text { with } p \\
\text { refinement }\end{array}$} \\
\hline & \multirow[b]{2}{*}{ Obs. } & \multirow[b]{2}{*}{ CASC2D } & \multicolumn{2}{|c|}{ DG-SAKE } & \multirow[b]{2}{*}{ CASC2D } & \multicolumn{2}{|c|}{ DG-SAKE } & \\
\hline & & & $p=0$ & $p=2$ & & $p=0$ & $p=2$ & \\
\hline 1 & 7.06 & 7.01 & 7.72 & 7.85 & 0.05 & 0.66 & 0.79 & $\ldots$ \\
\hline 4 & 9.94 & 10.45 & 8.45 & 11.07 & 0.51 & 1.49 & 1.13 & 24.16 \\
\hline 6 & 11.71 & 8.62 & 8.79 & 11.96 & 3.09 & 2.92 & 0.25 & 91.44 \\
\hline 7 & 11.61 & 10.87 & 8.25 & 11.01 & 0.74 & 3.36 & 0.60 & 82.14 \\
\hline 8 & 17.07 & 8.50 & 10.57 & 14.71 & 8.57 & 6.50 & 2.36 & 63.69 \\
\hline 14 & 15.24 & 8.31 & 8.41 & 11.00 & 6.93 & 6.83 & 4.24 & 37.92 \\
\hline & & are & $\mathrm{e}$ & - & 3.32 & 3.63 & 1.56 & 57.02 \\
\hline
\end{tabular}




\section{Summary}

In this paper, we have presented the development and application of a multidimensional unstructured mesh model, called DG-SAKE, for simulating coupled overland/open-channel flows in the kinematic wave approximation regime. The modeling approach makes use of DG finite element spatial discretizations of variable degree $p$, paired with explicit RK time steppers, and is supported by advancements made to an automatic mesh generation tool, ADMESH+, previously developed by the first three authors. The developed model was applied to a set of four test cases, where numerical results were found to compare well with known analytic solutions, experimental data and results from a (structured, finite difference) model, CASC2D, that is well-established in this area of application. Of particular note, the numerical results obtained from DGSAKE demonstrate the potential benefits of using $p$ refinement, over standard $h$ (mesh) refinement, in this setting in order to achieve more accurate solutions in an efficient manner.

Future work on this particular model will focus on the development of a multi-physics approach within the model, whereby a numerical estimate of the kinematic wave (and Froude) numbers within each element will be computed and used to determine the appropriate set of governing equations, e.g., the kinematic wave approximations or the full shallow water equations, that should be solved in an element at a particular time. Among other advantages, this would allow, for example, the model to properly simulate so-called backwater effects that can occur in some flow scenarios. Finally, our future work in the general area of hydrological modeling will explore coupling the developed model to a DG-based subsurface flow model.

\section{Acknowledgment}

The authors acknowledge support by National Science Foundation grant DMS-1217218. 


\section{References}

[1] S. Fatichi, et al, An overview of current applications, challenges, and future trends in distributed process-based models in hydrology, Journal of Hydrology.

[2] C. Paniconi, M. Putti, Physically based modeling in catchment hydrology at 50: Survey and outlook, Water Resources Research 51 (2015) 7090-7129.

[3] W. Brutsaert, Hydrology: An introduction, Cambridge University Press, Cambridge, 2005.

[4] R. Biswas, K. D. Devine, J. Flaherty, Parallel, adaptive finite element methods for conservation laws, Applied Numerical Mathematics 14 (1994) 255283.

[5] E. J. Kubatko, S. Bunya, C. Dawson, J. J. Westerink, C. M. Mirabato, A performance comparison of continuous and discontinuous finite element shallow water models, Journal of Scientific Computing 40 (2009) 1573-7691.

[6] M. DuChene, A. M. Spagnuolo, E. J. Kubatko, J. Westerink, C. Dawson, A framework for running the ADCIRC discontinuous Galerkin storm surge model on a GPU, in: M. Sato, S. Matsuoka, P. M. A. Sloot, G. D. VanAlbada, J. Dongarra (Eds.), Proceedings of the International Conference on Computational Science (ICCS), Vol. 4 of Procedia Computer Science, 2011, pp. 2017-2026.

[7] M. Fuhry, A. Giuliani, L. Krivodonova, Discontinuous galerkin methods on graphics processing units for nonlinear hyperbolic conservation laws, International Journal for numerical methods in fluids 76 (12) (2014) 9821003.

[8] A. Klockner, T. Warburton, J. Bridge, J. S. Hesthaven, Nodal discontinuous Galerkin methods on graphics processors, Journal of Computational Physics 228 (21) (2009) 7863-7882. 
[9] C. Dawson, S. Y. Sun, M. F. Wheeler, Compatible algorithms for coupled flow and transport, Computer Methods in Applied Mechanics and Engineering 193 (23-26) (2004) 2565-2580.

[10] W. Brutsaert, Encyclopedia of Geomorphology, Vol. 1 \& 2, Routledge International Association of Geomorphologists, London, 2004.

[11] C. J. Conroy, E. J. Kubatko, D. W. West, ADMESH: an advanced, automatic unstructured mesh generator for shallow water models, Ocean Dynamics 62 (10) (2012) 1503-1517.

[12] J. H. D. Vieira, Conditions governing the use of approximations for the Saint-Venant equations for shallow surface-water flow, Journal of Hydrology $60(1-4)(1983) 43-58$.

[13] D. A. Woolhiser, J. A. Liggett, Unsteady, one-dimensional flow over a plane — the rising hydrograph, Water Resources Research 3 (3) (1967) 753-771.

[14] V. P. Singh, Is hydrology kinematic?, Hydrological Processes 16 (2002) $667-716$.

[15] Y. Cheng, F. Li, J. Qiu, L. Xu, Positivity-preserving DG and central DG methods for ideal MHD equations, Journal of Computational Physics 238 (2013) 255-280.

[16] E. J. Kubatko, J. J. Westerink, C. Dawson, $h p$ discontinuous Galerkin methods for advection dominated problems in shallow water flow, Computer Methods in Applied Mechanics and Engineering 196 (2006) 437-451.

[17] C. Mirabito, C. Dawson, E. J. Kubatko, J. J. Westerink, S. Bunya, Implementation of a discontinuous Galerkin morphological model on twodimensional unstructured meshes, Computer Methods in Applied Mechanics and Engineering 200 (2011) 189-207.

[18] C. J. Trahan, C. Dawson, Local time-stepping in Runge-Kutta discontinuous Galerkin finite element methods applied to the shallow-water equations, 
Computer Methods in Applied Mechanics and Engineering 217 (2012) 139152.

[19] Y. Xing, X. Zhang, C.-W. Shu, Positivity-preserving high order wellbalanced discontinuous Galerkin methods for the shallow water equations, Advances in Water Resources 33 (2010) 1476-1493.

[20] V. Chow, Open-channel Hydraulics, McGraw-Hill, 1973.

[21] Q. Liu, L. Chen, J. Li, V. Singh, Two-dimensional kinematic wave model of overland-flow, Journal of Hydrology 291 (2004) 28-41.

[22] L. R. Foulds, Graph theory applications, Springer-Verlag, New York, 1992.

[23] G. E. Karniadakis, S. J. Sherwin, Spectral/hp element methods for CFD, Oxford University Press, New York, 1999.

[24] B. Cockburn, C.-W. Shu, Runge-Kutta discontinuous Galerkin methods for convection-dominated problems, Journal of Scientific Computing 16 (3) (2001) 173-261.

[25] S. Gottlieb, D. Ketcheson, C.-W. Shu, Strong Stability Preserving RungeKutta and Multistep Time Discretizations, World Scientific, 2011.

[26] W. Schwanghart, D. Scherler, Short Communication: Topotoolbox $2-$ an efficient and user-friendly tool for Earth surface sciences, Earth Surface Dynamics Discussions 1 (1) (2013) 261-275.

[27] P. O. Persson, G. Strang, A simple mesh generator in Matlab, SIAM Review 46 (2) (2004) 329-345.

[28] J. Hobby, Smooth, easy to compute interpolating splines, Discrete and Computational Geometry 1 (1986) 123-140.

[29] V. P. Singh, Kinematic Wave Modeling in Water Resources: Surface Water Hydrology, John Wiley \& Sons, New York, NY, 1997. 
[30] V. P. Singh, Effect of the direction of storm movement on planar flow, Hydrological Processes 12 (1998) 147-170.

[31] V. P. Singh, Effect of the duration and direction of storm movement on infiltrating planar flow with full areal coverage, Hydrological Processes 16 (2002) 1479-1511.

[32] D. W. West, A multidimensional discontinuous Galerkin modeling framework for overland flow and channel routing, Master's thesis, The Ohio State University (2015).

[33] C. M. Kazezyilmaz-Alhan, M. A. M. Jr., P. Rao, On numerical modeling of overland flow, Applied Mathematics and Computation 166 (2005) 724-740.

[34] J. Kim, A. Warnock, V. Y. Ivanov, N. D. Katopodes, Coupled modeling of hydrologic and hydrodynamic processes including overland and channel flow, Advances in Water Resources 37 (2012) 104-126.

[35] C. Yu, J. Duan, Two-dimensional hydrodynamic model for surface-flow routing, Journal of Hydraulic Engineering 140 (9) (2014) 04014045.

[36] R. A. Wooding, A hydraulic model for the catchment-stream problem: I. kinematic-wave theory, Journal of Hydrology 3 (3-4) (1965) 254-267.

[37] Y. Iwagaki, Fundamental studies of runoff analysis by characteristics, Bulletin 10, Disaster Prevention Research Institute, Kyoto University, Kyoto, Japan, 25pp (1955).

[38] K. Feng, F. J. Molz, A 2-D diffusion-based, wetland flow model, Journal of Hydrology 196 (1-4) (1997) 230-250.

[39] M. Santillana, C. Dawson, A numerical approach to study the properties of solutions of the diffusive wave approximation of the shallow water equations, Computational Geosciences 14 (1) (2010) 31-53.

[40] W. Zhang, T. W. Cundy, Modeling of two-dimensional overland flow, Water Resources Research 25 (9) (1989) 2019-2035. 
[41] E. M. Morris, D. A. Woolhiser, Unsteady one-dimensional flow over a plane: partial equilibrium and recession hydrographs, Water Resources Research 16 (2) (1980) 355-360.

[42] W. A. Blackmarr, Documentation of hydrologic, geomorphic, and sediment transport measurements on the Goodwin Creek experimental watershed, northern Mississippi, for the period 1982-1993-preliminary release, Research Report 3, U.S. Dept. of Agriculture, Agricultural Research Service (1995).

[43] T. W. Sturm, Open Channel Hydraulics, 2nd Edition, McGraw-Hill, 2010.

[44] The CASC2D-SED watersed model, http://www.engr.colostate. edu/ pierre/ce_old/Projects/CASC2D-SED\%20Web\%20site\%20082506/ CASC2D-SED-Home.htm, accessed: 2015-03-02.

[45] R. R. Sánchez, GIS-based upland erosion modeling, geovisualization and grid size effects on erosion simulations with CASC2D-SED, Ph.D. thesis, Colorado State University (2002).

[46] J. Singh, M. S. Altinakar, Y. Ding, Numerical modeling of rainfallgenerated overland flow using nonlinear shallow-water equations, Journal of Hydrologic Engineering 20 (8) (2015) 04014089.

[47] R. Rojas, P. Julien, B. Johnson, A 2-Dimensional Rainfall-Runoff and Sediment Model, Colorado State University (June 2003). 